\title{
AIF loss deregulates hematopoiesis and reveals different adaptive metabolic responses in bone marrow cells and thymocytes
}

\author{
Lauriane Cabon ${ }^{1,2} \cdot$ Audrey Bertaux $^{1,2} \cdot$ Marie-Noëlle Brunelle-Navas $^{1,2} \cdot$ Ivan Nemazanyy ${ }^{3}$ - Laurianne Scourzic ${ }^{4}$. \\ Laure Delavallée $^{1,2} \cdot$ Laura Vela $^{1,2} \cdot$ Mathieu Baritaud ${ }^{1,2} \cdot$ Sandrine Bouchet ${ }^{1,2} \cdot$ Cécile Lopez $^{4} \cdot$ Vu Quang Van $^{5}$. \\ Kevin Garbin ${ }^{2,6} \cdot$ Danielle Chateau $^{2,6} \cdot$ Françoise Gilard $^{7} \cdot$ Marika Sarfati $^{5} \cdot$ Thomas Mercher $^{4} \cdot$ Olivier A. Bernard ${ }^{4}$. \\ Santos A. Susin $\mathbb{D}^{1,2}$
}

Received: 5 April 2017 / Revised: 21 September 2017 / Accepted: 16 October 2017 / Published online: 11 January 2018

(c) ADMC Associazione Differenziamento e Morte Cellulare 2018

\begin{abstract}
Mitochondrial metabolism is a tightly regulated process that plays a central role throughout the lifespan of hematopoietic cells. Herein, we analyze the consequences of the mitochondrial oxidative phosphorylation (OXPHOS)/metabolism disorder associated with the cellspecific hematopoietic ablation of apoptosis-inducing factor (AIF). AIF-null $\left(A I F^{-1 Y}\right.$ ) mice developed pancytopenia that was associated with hypocellular bone marrow (BM) and thymus atrophy. Although myeloid cells were relatively spared, the B-cell and erythroid lineages were altered with increased frequencies of precursor B cells, pro-erythroblasts I, and basophilic erythroblasts II. T-cell populations were dramatically reduced with a thymopoiesis blockade at a double negative (DN) immature state, with DN1 accumulation and delayed DN2/DN3 and DN3/DN4 transitions. In BM cells, the OXPHOS/metabolism dysfunction provoked by the loss of AIF was counterbalanced by the augmentation of the mitochondrial biogenesis and a shift towards anaerobic glycolysis. Nevertheless, in a caspase-independent process, the resulting excess of reactive oxygen species compromised the viability of the hematopoietic stem cells (HSC) and progenitors. This led to the progressive exhaustion of the HSC pool, a reduced capacity of the BM progenitors to differentiate into colonies in methylcellulose assays, and the absence of cell-autonomous HSC repopulating potential in vivo. In contrast to BM cells, $A I F^{-/ Y}$ thymocytes compensated for the OXPHOS breakdown by enhancing fatty acid $\beta$-oxidation. By over-expressing CPT1, ACADL and PDK4, three key enzymes facilitating fatty acid $\beta$-oxidation (e.g., palmitic acid assimilation), the $A I F^{-/ Y}$ thymocytes retrieved the ATP levels of the $A I F^{+/ Y}$ cells. As a consequence, it was possible to significantly reestablish $A I F^{-/ Y}$ thymopoiesis in vivo by feeding the animals with a high-fat diet complemented with an antioxidant. Overall, our data reveal that the mitochondrial signals regulated by AIF are critical to hematopoietic decision-making. Emerging as a link between mitochondrial metabolism and hematopoietic cell fate, AIFmediated OXPHOS regulation represents a target for the development of new immunomodulatory therapeutics.
\end{abstract}

\section{Introduction}

Mitochondria are functional organelles that play a vital role during hematopoiesis by providing energy and by

Lauriane Cabon and Audrey Bertaux share first authorship.

Marie-Noëlle Brunelle-Navas, Ivan Nemazanyy and Laurianne Scourzic contributed equally to this work.

Edited by A. Villunger

Electronic supplementary material The online version of this article (https://doi.org/10.1038/s41418-017-0035-x) contains supplementary material, which is available to authorized users.

Santos A. Susin

santos.susin@crc.jussieu.fr

Extended author information available on the last page of the article generating reactive oxygen species (ROS) that regulate proliferation, differentiation, and self-renewal [1-4]. For example, a fine regulation of mitochondrial ROS production is needed to maintain the equilibrium between quiescent and cycling bone marrow (BM) hematopoietic stem cells (HSC) [2, 5-7]. In contrast, high levels of mitochondrial ROS are detrimental to HSC and can lead to their elimination by programmed cell death [7]. The relevance of mitochondrial oxidative phosphorylation (OXPHOS) in determining HSC fate is also signified by the metabolic changes that occur during the transition from a quiescent to a cycling/activated state [8]. Concerning thymopoiesis, less is known about the OXPHOS implication in the differentiation of double negative (DN) progenitor cells. However, a fine-tuning between ROS and glycolytic activation appears important for DN1-toDN4 transitions $[9,10]$. 
Apoptosis-inducing factor (AIF), a Janus mitochondrial protein that is encoded by the Aifml (Aif, Pdcd8) gene on the $\mathrm{X}$-chromosome, has been implicated in maintaining mitochondrial electron transport chain (ETC) function [11-14]. After its translocation to the nucleus following an apoptotic insult, AIF also participates in the modulation of caspase-independent programmed cell death [15-21]. Under pathological conditions, the mitochondrial function of AIF is critical to inhibiting tumor metastasis by modulating PTEN phosphatase activity [22]. Moreover, human mutations in AIF result in acute pediatric mitochondriopathies [23-25]. Concerning the immune system, studies in the Harlequin (Hq) mouse strain (which has a $\sim 70 \%$ reduction in AIF expression) indicate that, in peripheral mature $\mathrm{T}$ cells, low AIF expression leads to enhanced susceptibility of T-cell blasts to activation-induced cell death and reduced sensitivity to neglect-induced cell death. In this model, the HSC, B cells and other hematopoietic lineages do not exhibit apparent abnormalities [26, 27]. Contrary to these results, a work in which Aif was ablated around the DN3 stage of thymocyte development reported that AIF loss had no impact on T-cell development. In the same study, the use of the CD19-cre strain indicated that AIF was not required for B-cell development and function [28].

Herein, by generating an original mouse model in which Aif was ablated early during hematopoiesis, we have broadly investigated the role of AIF and the OXPHOS metabolism in the development of immune cells. Our mouse strain demonstrates that early AIF deficiency provokes pleiotropic consequences on normal hematopoiesis, the arrest of thymopoiesis, and a delayed development of B-cell and erythroid lineages. Importantly enough, our mouse model also illustrates metabolic adaptive responses associated to OXPHOS dysfunction: BM cells shift towards anaerobic glycolysis, whereas thymocytes favor fatty acid $\beta$-oxidation (FAO).

\section{Results}

\section{AIF ablation in hematopoietic cells resulted in pancytopenia and aberrant thymocyte, B-cell, and erythroid development}

To investigate the influence of AIF in hematopoiesis, we crossed a newly generated Aifm1-floxed mouse with a Vavl-cre strain that drives early Cre recombinase expression specifically in hematopoietic cells, including HSC [29]. Excision of Aifml exon 11 on the X-chromosome generated a stop codon, which resulted in a male progeny null for AIF $\left(A I F^{-/ Y}\right.$ mice) (Supplementary Figure 1).

At 7 days of age, $A I F^{-Y}$ and $A I F^{+/ Y}$ littermates (WT mice) presented a similar phenotype with a normal BM cellularity. However, in contrast to $A I F^{+/ Y}$, the thymus of $\mathrm{KO}$ animals was markedly smaller (Fig. 1a). The $A I F^{-/ Y}$ newborns developed normally until they were 21 days old, and after that they presented signs of severe anemia (Fig. 1a, 28-day-old mice pictures), which was followed by mice death (Fig. 1b). White cells, red cells, and platelet counts confirmed that $A I F^{-/ Y}$ mice displayed progressive pancytopenia (Fig. 1c). Between 21 and 28 days after birth, $A I F^{-/ Y}$ animals exhibited severe hypocellularity of the BM and spleen. Moreover, the number of thymocytes in these animals, close to zero at birth, did not increase with age. Finally, the lymph node cellularity was very low in the $\mathrm{KO}$ mice, with no observed evolution and even a complete collapse in the 28-day-old animals (Fig. 1d).

To better analyze the hematopoietic lineages in $A I F^{+/ Y}$ and $A I F^{-Y}$ mice, we performed cell-specific immunophenotyping. In the BM, although no significant differences were recorded in 7-day-old mice, erythroid, macrophagic/ monocytic, B-lymphoid, and T-lymphoid cells were severely decreased in 21- and 28-day-old $A I F^{-/ Y}$ animals (Fig. 1e). In the thymus of the $\mathrm{KO}$ mice, T-cell development was blocked at the $\mathrm{CD}^{-} \mathrm{CD}^{-}$DN stage (Fig. 1f and Supplementary Figure 2). Our analysis of other hematopoietic lineages revealed that, in contrast to what had been previously reported in the Hq mouse strain [26, 27] or in the CD19-Cre mouse model [28], the B-cell and erythroid lineages were also affected by the early AIF deficiency. This was manifested by increased frequencies of precursor B cells in the BM and spleen (Fig. 1g and Supplementary Table 1), as well as increased frequencies of pro-erythroblasts I and basophilic erythroblasts II in the spleen (Fig. 1h).

By crossing Aifml-floxed mice with a tamoxifen inducible Rosa26-CreERT2 mouse strain [30], we confirmed that the loss of AIF in 3-month-old adult mice also provoked major hematopoietic alterations. These included a reduction in BM and thymus cellularity, which resulted in death of the mice between 25 and 65 days after the first tamoxifen injection, and a significant delay in T-cell development (Supplementary Figures 3a-c). Overall, these findings indicate that AIF ablation resulted in pleiotropic consequences that affected hematopoiesis in neonatal and adult mice.

\section{AIF loss provoked mitochondrial OXPHOS dysfunction and BM metabolic recalibration}

As indicated above, AIF has been implicated in maintaining ETC stability and function [12-14]. Thus, we tested whether the severe phenotype observed in $A I F^{-Y}$ mice was directly related to OXPHOS dysfunction in BM cells. ETC integrity 
a

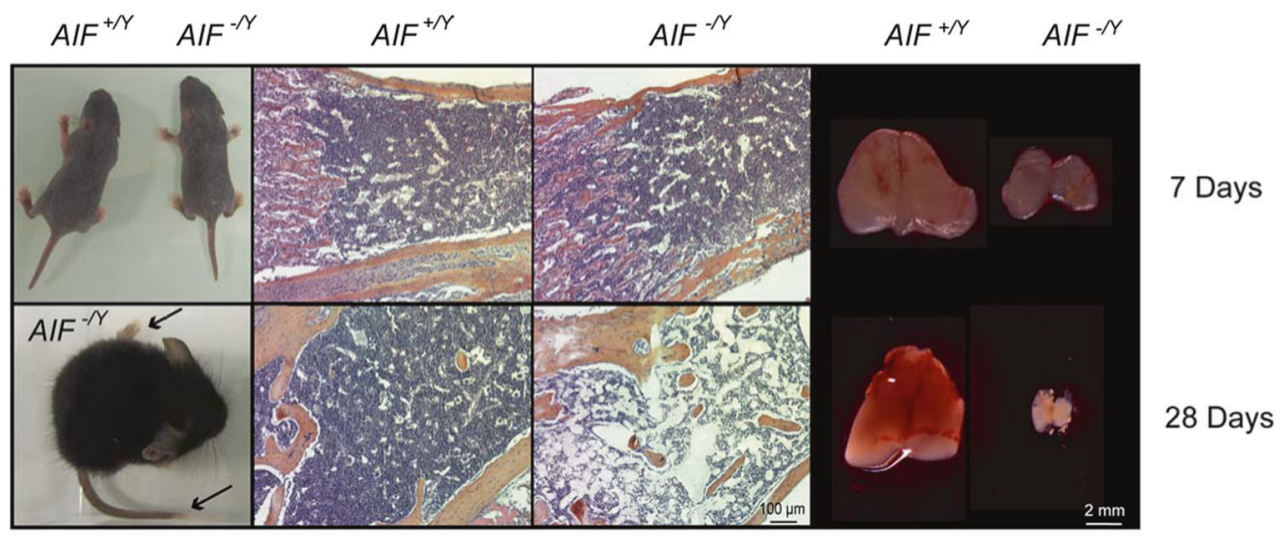

b

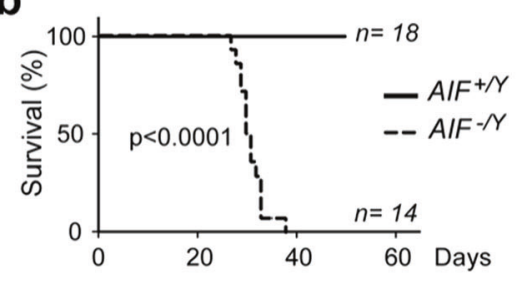

C

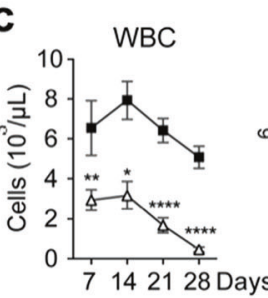

ys

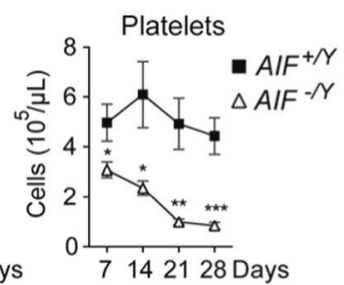

d
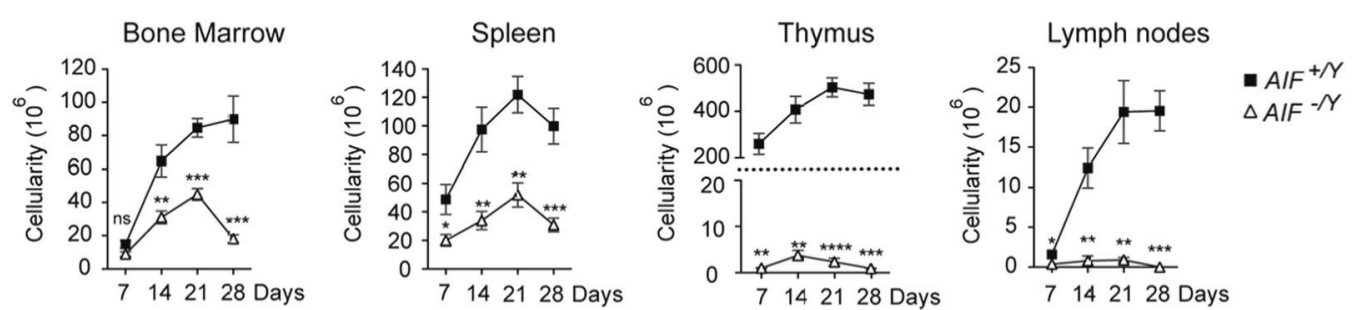

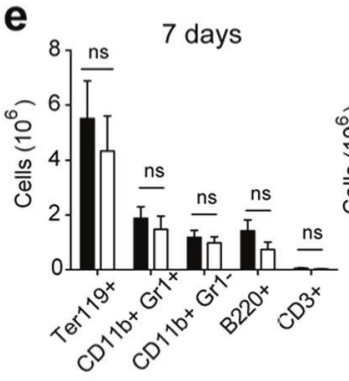

g

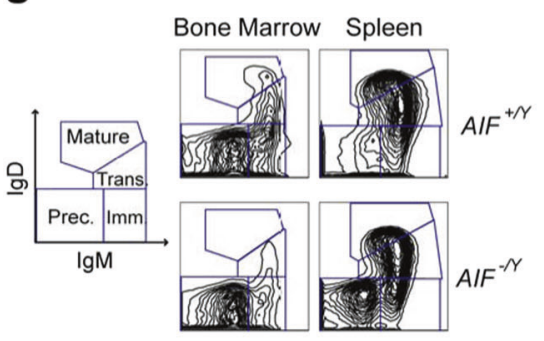

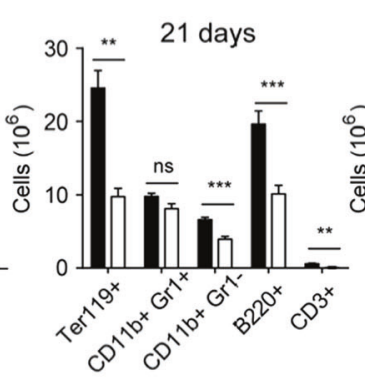

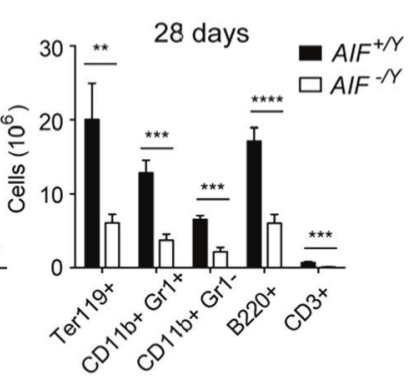

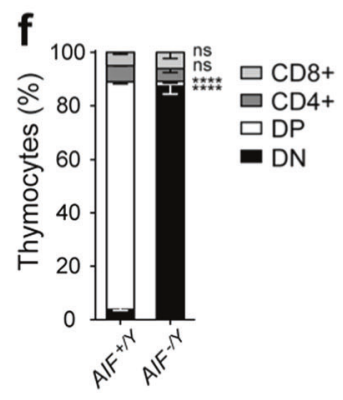

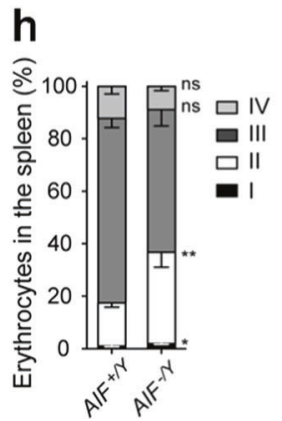


4 Fig. 1 AIF loss led to pancytopenia and hematopoietic developmental defects. a Left, representative images of $A I F^{+/ Y}$ and $A I F^{-/ Y}$ mice. $A I F^{-/ Y}$ 28-day-old animals present external signs of anemia in fingers and tail (arrows). Middle and right, H/E staining of BM and overall thymus size observed in $A I F^{+/ Y}$ and $A I F^{-/ Y}$ mice at the indicated time after birth. b Kaplan-Meier survival probability of $A I F^{+Y}$ and $A I F^{-/ Y}$ mice. c Kinetic cell count of white blood cells (WBC), red blood cells (RBC), and platelets from $A I F^{+Y Y}$ and $A I F^{-/ Y}$ mice $(n=10$ mice per group). d Kinetic assessment of cellularity in BM, spleen, thymus, and lymph nodes from $A I F^{+/ Y}$ and $A I F^{-/ Y}$ mice $(n=10$ mice per group). e Number of BM erythroid (Ter1 $\left.19^{+}\right)$, granulocytic $\left(\mathrm{CD} 11 \mathrm{~b}^{+} \mathrm{Gr}^{+}\right)$, macrophagic/monocytic $\left(\mathrm{CD} 11 \mathrm{~b}^{+} \mathrm{Gr}^{-}\right)$, B cells $\left(\mathrm{B} 220^{+}\right)$, and $\mathrm{T}$ cells $\left(\mathrm{CD} 3^{+}\right)$measured in 7 - to 28-day-old $A I F^{+/ Y}$ and $A I F^{-/ Y}$ mice ( $n=8$ mice per group). $\mathbf{f}$ Percentages of $\mathrm{CD} 4^{-} / \mathrm{CD} 8^{-}$(DN), $\mathrm{CD}^{+} / \mathrm{CD}^{+}$(DP), CD4 ${ }^{+}$, and CD8 ${ }^{+}$thymocytes recorded in 21-day-old $A I F^{+/ Y}$ and $A I F^{-/ Y}$ mice $(n=10$ mice per group). $\mathrm{g}$ Left, flow cytometry panels of $\operatorname{IgD} / \operatorname{IgM}$ staining on B220-expressing $\mathrm{BM}$ and spleen cells from 21 day-old $A I F^{+/ Y}$ and $A I F^{-/ Y}$ animals. Right, frequencies of precursor $\left(\operatorname{IgM}^{-} \operatorname{IgD}^{-}\right)$, immature $\left(\operatorname{IgM}^{+} \operatorname{IgD}{ }^{-}\right)$, transitional $\left(\operatorname{IgM}^{+} \operatorname{IgD}{ }^{\text {int }}\right)$, and mature $\left(\operatorname{IgM}^{+} \operatorname{IgD}^{+}\right) \mathrm{B} 220^{+} \mathrm{B}$ cells from 21 -day-old $A I F^{+/ Y}$ and $A I F^{-/ Y}$ mice $(n=14$ mice per group). $\mathbf{h}$ Percentages of proerythroblasts I $\left(\mathrm{CD} 71^{+}\right.$Ter1 $\left.19^{\text {int }}\right)$, basophilic erythroblasts II $\left(\mathrm{CD} 71^{+}\right.$Ter $\left.119^{+}\right)$, late basophilic and chromatophilic erythroblasts III $\left(\mathrm{CD} 71^{\text {int }}\right.$ Ter1 19 ${ }^{+}$), and orthochromatic erythroblasts IV $\left(\mathrm{CD} 71^{-}\right.$Ter $\left.119^{+}\right)$assessed in 21-day-old $A I F^{+/ Y}$ and $A I F^{-/ Y}$ mice $(n=6$ mice per group). Statistical significance was calculated by Mann-Whitney test. Symbols and bars represent mean \pm SEM

was verified using an immunoblot approach, which indicated that the $A I F^{-1 Y} \mathrm{BM}$ cells experienced a reduction in the mitochondrial complex I NDUFA9, complex III UQCRC2, and complex IV COX4I2 subunits. By contrast, complex II SDHA and complex V ATP5B proteins were not affected by the AIF loss (Fig. 2a and Supplementary Figure 4a). These observations are in accordance with the "solid-state" model of ETC structure that organizes the mitochondrial complexes I, III, and IV in a unique "supercomplex" entity in which the alteration in one complex directly affects the two others [3133]. Note that the structural mitochondrial protein VDAC1 appears overexpressed in the BM KO cells, suggesting an augmentation of the mitochondrial mass. Morphologically, compared with WT cells, mitochondria from $A I F^{-Y Y}$ cells lacked a normal tubular structure and exhibited swollen irregular tubules associated with changes in the internal membrane organization (Fig. 2b).

The monitoring of oxygen consumption rate (OCR) revealed that $A I F^{-Y}$ cells exhibited a significant reduction in both basal oxygen consumption and total oxidative capacity (Fig. 2c and Supplementary Figure 4b). In spite of that, the complex I inhibitor amytal abolished the remaining oxygen consumption of $A I F^{-/ Y}$ cells, indicating that complex I remains somewhat functional in these cells. Moreover, as indicated by the addition of the complex $\mathrm{V}$ inhibitor oligomycin, electron transport was still coupled to ATP synthesis (Fig. 2c and Supplementary Figure 4b). Finally, the detailed analysis of the individual complex activities, measured by monitoring OCR in permeabilized cells, revealed that ETC activities of $A I F^{-I Y}$ cells were $\sim 40 \%$ lower than those measured in WT cells (Supplementary Figure 4c). Together, the OXPHOS alterations associated to AIF deficiency in BM cells provoked a diminution in the mitochondrial transmembrane potential $(\Delta \Psi \mathrm{m})$ and an enhancement of mitochondrial ROS (Fig. 2d and e and Supplementary Figures 3d and e).

At the cellular level, the results obtained confirm that the mitochondrial defects associated with AIF loss were counterbalanced in BM cells by the augmentation of mitochondrial mass (Fig. 2f). This was accompanied by a shift to anaerobic glycolysis, as revealed by the increase in: (i) glucose uptake (Fig. 2g); (ii) extracellular acidification rate (ECAR) (Fig. 2h); and (iii) lactate release (Fig. 2i). The inhibition of caspases does not modify this glycolytic picture (Supplementary Figure 5), indicating that the metabolic change is not owing to the initiation of an apoptotic process. Anyhow, the metabolic reprogramming was further validated by a broad metabolomic approach, which corroborated that the components of the glycolytic pathway and the general carbohydrate metabolism were enhanced in $A I F^{-/ Y}$ BM cells. The metabolomic approach also revealed that AIF-null BM cells had reinforced amino-acid metabolic pathways (Fig. 2j and Supplementary Table 2). However, the OCR monitoring of the major amino-acid substrate, glutamine, indicated that the BM cells do not use this pathway as a source of energy (Supplementary Figure 6a). The amino-acid increase seems to reflect here the low ability of AIF KO BM cells to proliferate, which could result in a general amino-acidic accumulation. Thus, the glycolytic shift seems sufficient to maintain the ATP levels of the $A I F^{-1 Y}$ BM cells close to those measured in WT cells (Fig. 2k and Supplementary Figure $3 \mathrm{f}$ and $6 \mathrm{~b}$ ). Overall, our data reveal that the loss of AIF in BM cells results in a defective OXPHOS that is counterbalanced by the stimulation of mitochondrial biogenesis and a metabolic shift that reinforces anaerobic glycolysis.

\section{Mitochondrial OXPHOS and metabolism defects associated with the absence of AIF modify the repopulating potential of BM hematopoietic progenitors}

$A I F^{-/ Y}$ animals displayed progressive pancytopenia that affected all hematopoietic subsets. This finding suggested that AIF was required to control HSC homeostasis. To confirm the role of AIF in hematopoietic progenitors, we first investigated the HSC-enriched $\mathrm{Lin}^{-} \mathrm{Sca}-{ }^{+} \mathrm{Kit}^{+}$ (LSK) fraction in both E17.5 embryos and 7- to 28-day-old animals. Compared with WT mice, AIF-deficient LSK cells markedly declined between 7 and 14 days after birth (Fig. 3a). In addition, analysis of HSC (LT-HSC, STHSC), multipotent progenitors (MPP), common lymphoid progenitors (CLP), myeloid progenitors (CMP and GMP) and megakaryocyte/erythrocyte progenitors (MEP) showed that the loss of cells affected all HSC and 
a

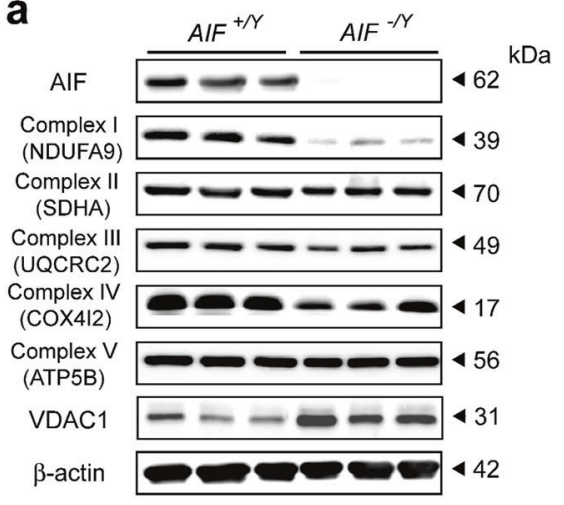

b

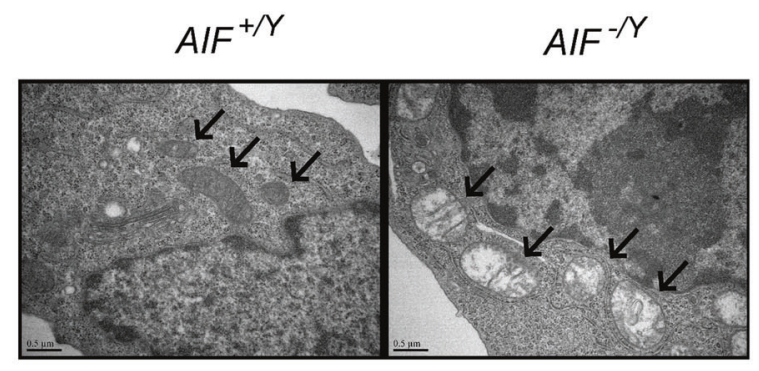

C

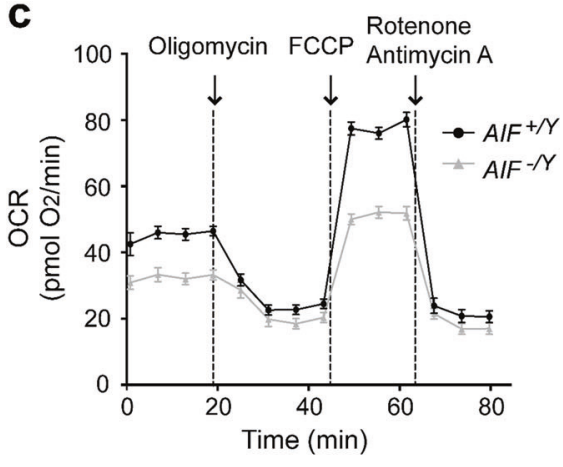

e

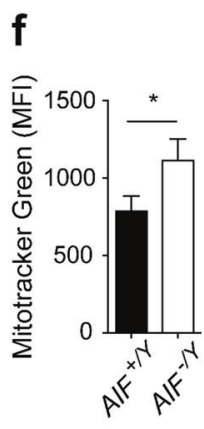

i

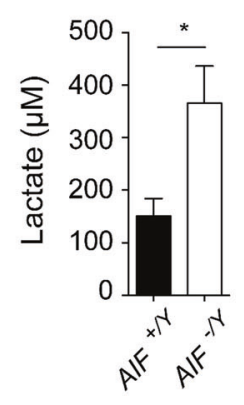

d

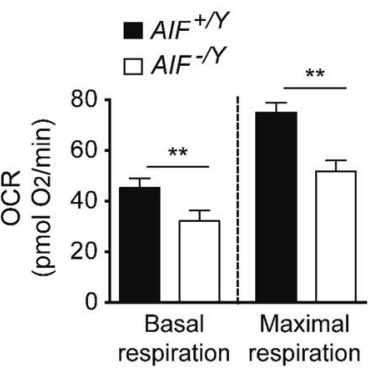

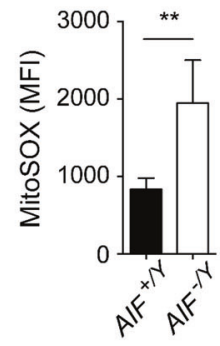

g

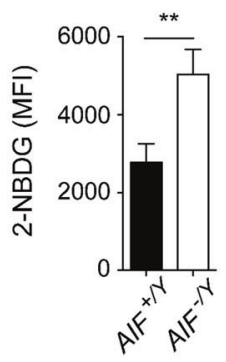

h

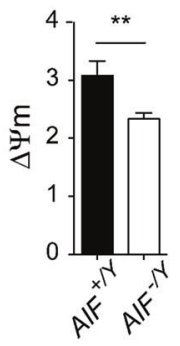

$$
\int^{4}
$$


Fig. 2 AIF loss induced OXPHOS dysfunction, ROS generation, and broad metabolic adjustments in BM cells. a Representative immunoblot of BM cells obtained from $A I F^{+/ Y}$ and $A I F^{-/ Y}$ 21-day-old animals revealing AIF and key proteins of the ETC complexes. VDAC1 was used as mitochondrial loading marker. Equal cellular loading was confirmed by $\beta$-actin probing ( $n=3$ independent experiments with similar results). b Representative electron micrographs of $A I F^{+/ Y}$ and $A I F^{-/ Y}$ BM cells. The black arrows mark the mitochondria. Bar: $0.5 \mu \mathrm{m}$. c Left, OCR of $A I F^{+/ Y}$ and $A I F^{-I Y} \mathrm{BM}$ cells under basal conditions (initial rates) and in response to sequential treatment with Oligomycin, FCCP, and Rotenone/ Antimycin A. Arrows indicate the time of the addition of each reagent. Right, Basal and maximal OCR of BM cells expressed as a histogram $(n=$ 3 independent experiments). $\mathbf{d} \Delta \Psi \mathrm{m}$ assessment performed in $A I F^{+/ Y}$ and $A I F^{-/ Y} \mathrm{BM}$ cells $(n=8$ mice per group). e Mitochondrial ROS levels recorded in $A I F^{+/ Y}$ and $A I F^{-I Y} \mathrm{BM}$ cells $\left(n=8\right.$ mice per group). $\mathbf{f}$ Left, Mitochondrial mass of $A I F^{+Y}$ and $A I F^{-/ Y}$ BM cells assessed by Mitotracker Green labeling ( $n=8$ mice per group). Right, PGCla mRNA levels determined by qPCR in $A I F^{+/ Y}$ and $A I F^{-/ Y} \mathrm{BM}$ cells $(n=5)$. The $18 \mathrm{~S}$ mRNA expression was used to normalize data. $\mathbf{g}$ Glucose uptake measured by the assimilation of 2-NBDG in $A I F^{+/ Y}$ and $A I F^{-/ Y}$ BM cells $(n=10$ mice per group). $\mathbf{h}$ Left, ECAR of $A I F^{+/ Y}$ and $A I F^{-/ Y} \mathrm{BM}$ cells measured in response to sequential addition of Glucose, Oligomycin, and 2-deoxyglucose (2-DG). Arrows indicate the time of the addition of each reagent. Right, ECAR of BM cells after Glucose treatment expressed as a histogram $(n=$ 3 independent experiments). i Lactate release analyzed in $A I F^{+Y Y}$ and $A I F^{-Y Y} \mathrm{BM}$ cells ( $n=7$ mice per group). $\mathbf{j}$ Heat map showing relative levels and hierarchical clustering of the indicated metabolite detected in BM cells. Each column corresponds to a different $A I F^{+/ Y}$ or $A I F^{-/ Y}$ animal and the color of the cell indicates the relative content of the metabolite (from green to red). Upon comparison with $A I F^{+/ Y}$ cells, only metabolites showing a significant modification in the $A I F^{-I Y}$ cells were represented. $\mathbf{k}$ ATP levels recorded in $A I F^{+/ Y}$ and $A I F^{-/ Y}$ BM cells $(n=8$ mice per group). Statistical significance was calculated by Mann-Whitney test. Bars represent mean \pm SEM. All tests were performed in 21-day-old animals

progenitor subsets. At the end, these subsets were nearly undetectable (Fig. 3b, 28-day-old animals). The LT-HSC, ST-HSC, and MPP subpopulations were also significantly affected in adult tamoxifen-treated $A I F^{-/ Y}$ mice (Supplementary Figure 3g).

We next assessed the functional properties of BM progenitors using ex vivo colony-forming assays. Compared with WT cells, the fetal liver (FL) KO cells obtained at E15.5 or BM cells from $A I F^{-/ Y}$ animals at 7 or 21 days of age had very low clonogenic capacity (Fig. 3c). Moreover, the colonies formed by all $\mathrm{KO}$ cells, which were significantly smaller than those generated by WT cells, were mainly derived from cells with an erythroid, granulocyte, or monocytic phenotype. At last, we demonstrated that the retroviral reintroduction of mAIF in KO cells significantly restored the clonogenic potential of $A I F^{-Y Y}$ cells (Fig. 3d). Overall, our colony-forming approaches confirm that the proliferation and differentiation defects of these $\mathrm{KO}$ cells result from the AIF absence.

We then performed both non-competitive and competitive transplantation assays to test the hematopoietic progenitor-repopulating capacities in vivo using CD45.2 donor cells that we injected into lethally irradiated CD45.1 recipient mice. In non-competitive assays, $\sim 50 \%$ of recipient mice grafted with $A I F^{-/ Y} \mathrm{BM}$ cells died. The surviving mice had very few CD45.2 donor cells in the blood or BM, as revealed by quantification at 2 , 6, and 8 weeks post transplantation (Fig. 3e). Similarly, in competitive transplantation experiments, AIF-deficient LSK cells yielded lower levels of reconstitution compared with WT LSK cells (Fig. 3f). Finally, we tested in vivo the repopulating capacities of the $A I F^{-/ Y}$ cells transduced with mAIF. As depicted in the Fig. $3 \mathrm{~g}$, the reintroduction of mAIF into $A I F^{-/ Y}$ FL cells significantly rescued their repopulating potential in vivo, confirming the cell-autonomous AIF requirement in HSC and progenitor cells.

\section{High levels of ROS induced AIF ${ }^{-/ Y}$ HSC and progenitors exhaustion}

We next investigated the mechanisms that underlie AIFmediated HSC and progenitors exhaustion. We first observed that anaerobic glycolysis is enhanced in these AIF KO subsets (Fig. 4a). Moreover, compared with WT cells, mitochondrial ROS levels were augmented (Fig. 4b). Finally, $A I F^{-/ Y} \mathrm{HSC}$ and progenitors presented increased frequencies of DNA damage and cell death (Fig. 4c-d).

Using the antioxidant $N$-acetyl-cysteine (NAC), we verified whether the levels of ROS generated by the AIF lossassociated OXPHOS dysfunction (Fig. 2) compromised the viability of $\mathrm{HSC}$ and progenitors. We first confirmed that treatment of $A I F^{-Y} \mathrm{BM}$ cells with NAC suppressed the excess of mitochondrial ROS (Fig. 4e). Further, using a colonyforming assay, we observed that the addition of NAC favored the development of $A I F^{-/ Y}$ BM cells into colonies (Fig. 4f). Notably, in the presence of NAC, $A I F^{-/ Y} \mathrm{BM}$ cells also developed into a large number of immature GEMM colonies, which would have never formed in the absence of antioxidant. Finally, the addition of NAC in the drinking water administered to the dams allowed for the in vivo rescue of LT-HSC, ST-HSC, and MPP in the $A I F^{-Y Y}$ newborns (Fig. 4g).

\section{Mitochondrial OXPHOS regulated thymocyte differentiation and fate}

To analyze the developmental defaults of the $A I F^{-/ Y}$ thymocytes, we first performed detailed immunophenotyping. In E15.5 thymic lobes, only DN precursor thymocytes were observed and an analysis of DN1-to-DN4 subpopulations did not reveal significant differences between the WT and KO animals (Fig. 5a and Supplementary Table 3a). At E17.5, loss of AIF restricted both the DN3-to-DN4 transition and the generation of DP cells. Furthermore, thymus analysis in 7, 21, and 28-day-old mice indicated that 

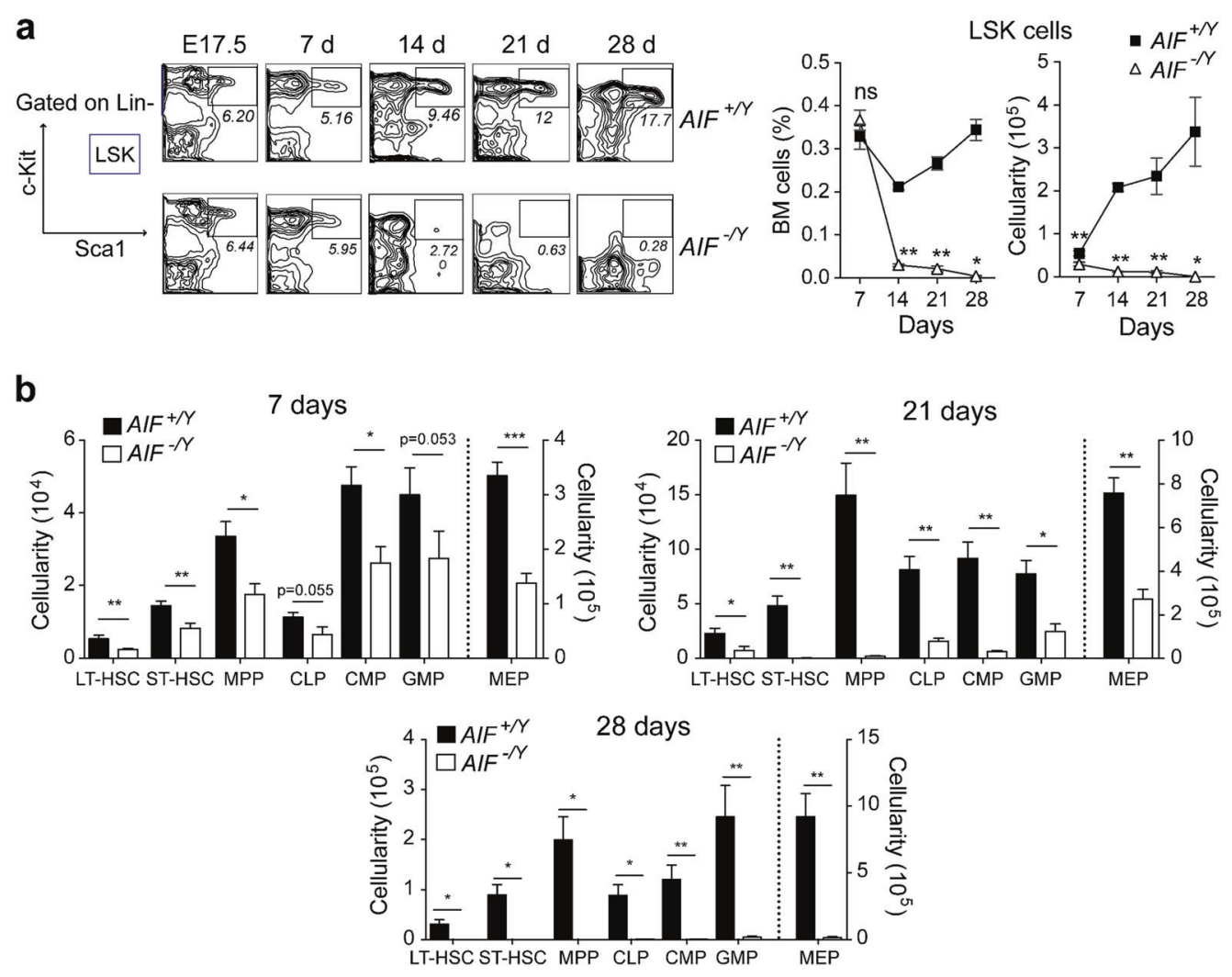

C
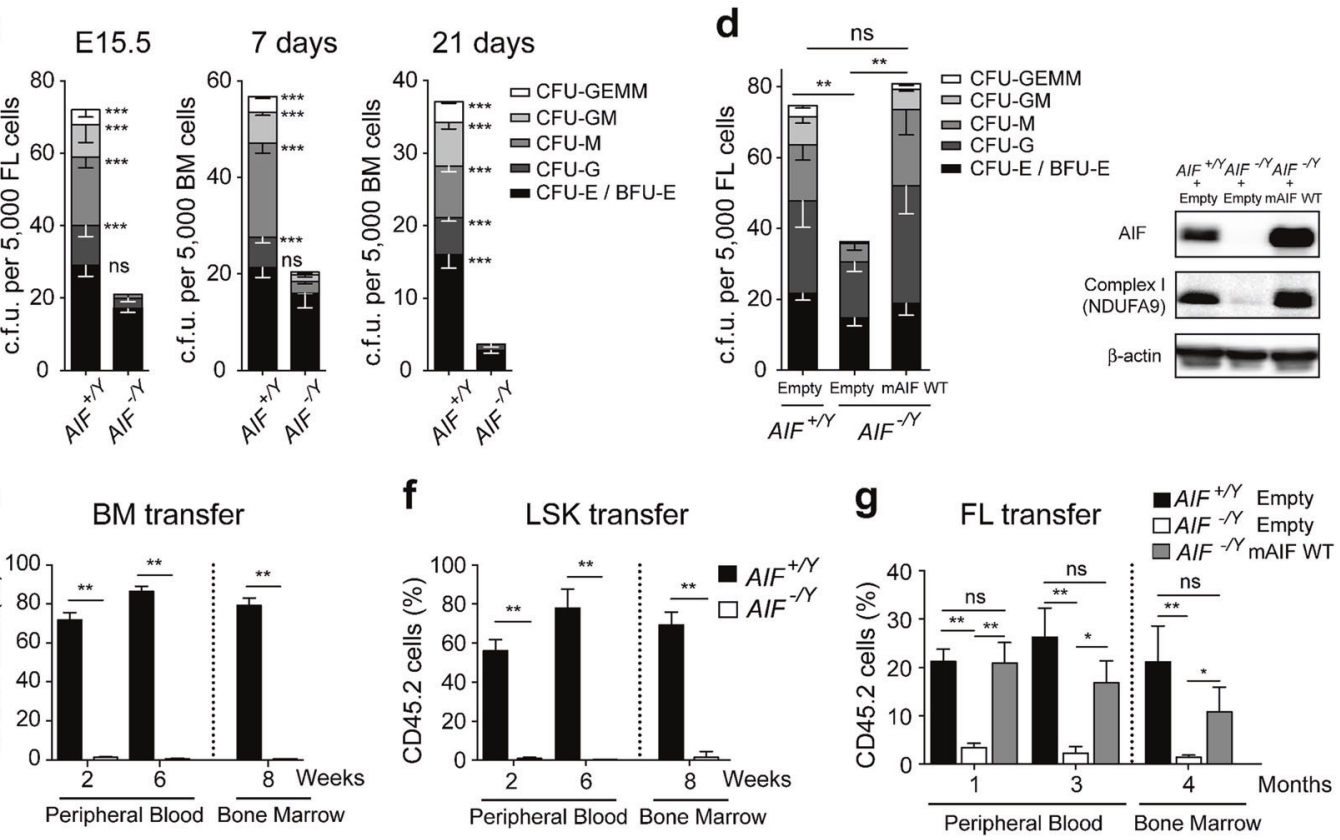

thymocytes from the $A I F^{-/ Y}$ progeny: (i) remained at a DN immature state; (ii) accumulated at the DN1 stage of differentiation; and (iii) exhibited delayed DN2/DN3 and DN3/DN4 transitions. In addition, cellular counts revealed a significant reduction in the DN2, DN3, and DN4 subsets in $A I F^{-/ Y}$ mice (Supplementary Figure 7). By using cKit/ CD24 co-labeling, we further analyzed the phenotype of the DN1 $A I F^{-/ Y}$ prothymocytes. Compared to WT, the DN1 compartment of the AIF-null 7-day-old mice showed a relative high number of DN1b cells, low levels of DN1c prothymocytes, and the accumulation of the less proliferative DN1e subset. In 21-day-old mice, as expected by the diminution of the CLP precursors in the BM (Fig. 3b), we detected an exhaustion of the canonical $\mathrm{T}$ progenitors 
Fig. 3 Mitochondrial OXPHOS dysfunction associated with AIF loss generated cell exhaustion and alteration in the repopulating potential of hematopoietic progenitors. a Left, Identification of LSK $\left(\mathrm{Lin}^{-} \mathrm{Sca}-1^{+} \mathrm{Kit}^{+}\right.$) cells in fetal liver (E17.5) or BM from 7-, 14-, 21-, and 28-day-old $A I F^{+Y Y}$ and $A I F^{-/ Y}$ animals by flow cytometry. Numbers are the percentages of LSK cells. Right, The number of LSK cells was recorded and graphed either as a percentage of total cells in the BM or as a number of cells (cellularity) ( $n=5$ mice per group). b Number of LT-HSC, ST-HSC, MPP, CLP, CMP, GMP, and MEP cells in 7- to 28-day-old animals ( $n=5$ mice per group). c Fetal liver cells (FL; E15.5) or BM cells from 7- or 21-day-old animals were seeded in methylcellulose culture and colonies formed by the GEMM, GM, or single lineages (G, M, and E) were counted ( $n=8$ independent experiments). $\mathbf{d}$ The effects of AIF re-expression on the colony-forming capacities were tested with E15.5 FL cells from $A I F^{+/ Y}$

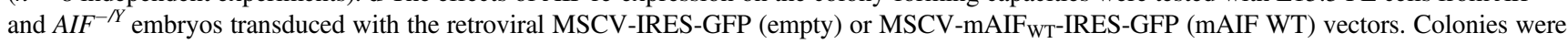
counted as in $\mathbf{c}$ ( $n=3$ independent experiments). The expression of AIF and the complex I subunit NDUFA9 was verified by immunoblotting. Equal loading was confirmed by $\beta$-actin probing. e In non-competitive transplantation experiments, CD45.2 donor BM cells from 7-day-old $A I F^{+/ Y}$ and $A I F^{-Y Y}$ mice were transplanted into irradiated CD45.1 recipient animals. CD45.2 chimerism in recipient mice was evaluated at the indicated time after transplantation ( $n=5$ mice per group). $\mathbf{f}$ In competitive transplantation experiments, CD45.2 donor LSK cells from 7-day-old WT or AIF KO animals were transplanted into irradiated CD45.1 mice along with recipient BM cells. CD45.2 chimerism in recipient mice was assessed at 2,6 , and 8 weeks after transplantation ( $n=5$ mice per group). $\mathbf{g}$ The effects of mAIF re-expression on hematopoietic reconstitution were tested with E15.5 FL cells from $A I F^{+/ Y}$ and $A I F^{-/ Y}$ embryos transduced with the retroviral MSCV-IRES-GFP (Empty) or MSCV-mAIF WT $_{\text {-IRES-GFP }}$ (mAIF WT) vectors ( $n=6$ mice per group). Statistical significance was calculated by Mann-Whitney a, b, $, \mathbf{e}, \mathbf{e}, \mathbf{f}$, and $\mathbf{g}$ or two-way ANOVA d tests. Symbols and bars represent mean \pm SEM

DN1a/DN1b. This was accompanied by the increase in the percentage of DN1e cells (75\% of the total DN1 cells; Fig. 5b and Supplementary Table 3b). Of note, the differences observed between the WT and $A I F^{-/ Y}$ thymocytes did not appear to be caused by impaired TCR $\beta$ recombination (Fig. 5c). Overall, our findings indicated that the loss of AIF influenced thymocyte differentiation and fate.

As for BM cells, we next tested whether the $A I F^{-/ Y}$ undifferentiated thymocyte phenotype was directly related to the mitochondrial OXPHOS alteration. Morphologically, compared with WT thymocytes, $A I F^{-/ Y}$ mitochondria exhibited a severe internal membrane disorganization that was associated to the loss of normal tubular structure (Fig. 6a). OCR monitoring revealed that $A I F^{-/ Y}$ thymocytes exhibited a $\sim 50 \%$ reduction in both basal oxygen consumption and total oxidative capacity (Fig. 6b). Thus, compared with $A I F^{-/ Y} \mathrm{BM}$ cells, the $A I F^{-/ Y}$ thymocytes showed a more severe OXPHOS dysfunction that provoked a reduction in $\Delta \Psi \mathrm{m}$ and the high generation of mitochondrial ROS (Fig. 6c and d and Supplementary Figures $3 \mathrm{~h}$ and i). Surprisingly, contrary to BM cells, these mitochondrial alterations were not counterbalanced either by an increase in the mitochondrial mass or by a shift towards anaerobic glycolysis (Fig. 6e and f). Consequently, the cellular ATP levels of the $A I F^{-/ Y}$ thymocytes dropped significantly (Fig. $6 \mathrm{~g}$ and Supplementary Figure 3j).

Searching for a metabolic adaptive alternative in $A I F^{-/ Y}$ thymocytes, we performed a quantitative-PCR approach that revealed the upregulation of three key FAO-facilitating enzymes: CPT1, ACADL, and PDK4 (Fig. 7a). This overexpression was corroborated by RNAscope, a specific fluorescence in situ hybridization technique [34] (Fig. 7b). Note that the metabolic reprogramming induced by AIF loss in thymocytes, including the mitochondrial alterations, the low glycolytic rate, and the overexpression of FAOfacilitating enzymes, was confirmed in comparing the $A I F$
$+/ Y$ and $A I F^{-/ Y} \mathrm{DN}$ thymocyte fractions (Supplementary Figure 8). Next, we biochemically confirmed the capacity of KO thymocytes to perform FAO. Related to WT, we observed the increased respiratory capacity of the $A I F^{-/ Y}$ thymocytes in the presence of palmitate (Fig. 7c, left panel). In these conditions, the respiratory capacity of the $A I F^{-/ Y}$ thymocytes was downregulated by the CPT1 inhibitor Etomoxir, corroborating the participation of this enzyme in palmitate assimilation (Fig. 7c, right panel). Altogether, our data indicate that the OXPHOS dysfunction associated to AIF loss could be counterbalanced in thymocytes by FAO stimulation. As for BM cells, the inhibition of caspases does not modify this metabolic reprogramming (Supplementary Figure 9).

\section{The ablation of AIF differentiated the metabolic flexibility of thymocytes and BM cells}

Finally, following our above results and considering that a ketogenic diet has been shown to improve medical condition in children bearing ETC mutations [35], we tested the possibility of reactivating thymopoiesis in vivo by eliminating the excess of ROS and feeding the dams with a high-fat diet (HFD) in which the major source of energy changed from carbohydrates to fatty acids [36]. We first demonstrated that NAC treatment efficiently suppressed the excess of mitochondrial ROS in $A I F^{-/ Y}$ thymocytes (Fig. 7d). Moreover, the treatment of the dams with HFD and, more evidently, with NAC + HFD restored the normal generation of ATP in the $A I F^{-/ Y}$ thymocytes (Fig. 7e and Supplementary Table 4a). Strikingly, compared with that observed in animals fed in a standard carbon diet, thymocyte development was significantly reestablished in the AIF ${ }^{-} / Y$ newborns fed with a HFD: (i) HFD alone favored the generation of DP thymocytes (a mean of 16\%) and (ii) a HFD + NAC combination allowed for a highly significant DP development (a mean of $40 \%$ ) (Fig. 7f and Supplementary Table 4b). These results confirm in vivo the 
Fig. 4 High levels of ROS in the AIF $^{-1}$ HSC and progenitor cells provoked DNA damage and apoptosis. a Left, ECAR of AIF $+/ Y$ and $A I F^{-/ Y} \mathrm{HSC}$ and

progenitors cells measured in response to sequential addition of Glucose, Oligomycin, and 2deoxyglucose (2-DG). Arrows indicate the time of the addition of each reagent. Right, ECAR of HSC and progenitor cells after Glucose treatment expressed as a histogram ( $n=3$ independent experiments). b-d

Mitochondrial ROS generation (MitoSOX labeling) b, DNA damage ( $\gamma \mathrm{H} 2 \mathrm{AX}$ levels) $\mathbf{c}$, and cell death (Annexin-V/7AAD co-staining) d were evaluated in $A I F^{+/ Y}$ and $A I F^{-Y Y}$ LT-HSC, STHSC, MPP cells from 7-day-old mice. Data are the fold change recorded in the $A I F^{-I Y}$ cells relative to the basal levels of the $A I F^{+/ Y}$ cells $(n=5$ mice per group). e Mitochondrial ROS levels measured by flow cytometry in $A I F^{+/ Y}$ and $A I F^{-/ Y}$ $\mathrm{BM}$ cells in the presence or absence of NAC ( $n=6$ mice per group). $\mathbf{f}$ Colony counting of $\mathrm{BM} A I F^{+/ Y}$ or $A I F^{-/ Y}$ cells plated in methylcellulose in the presence or absence of NAC ( $n$ $=5$ independent experiments). Representative images of the morphology of the different colonies are shown. g Dams were supplied or not with NAC in drinking water and the number of LT-HSC, ST-HSC, and MPP cells from 21-day-old $A I F^{+/ Y}$ and $A I F^{-/ Y}$ newborn mice was counted ( $n=7$ mice per group). Statistical significance was calculated by Mann-Whitney (a-e and $\mathbf{g}$ or two-way ANOVA $\mathbf{f}$ tests. Bars represent mean \pm SEM

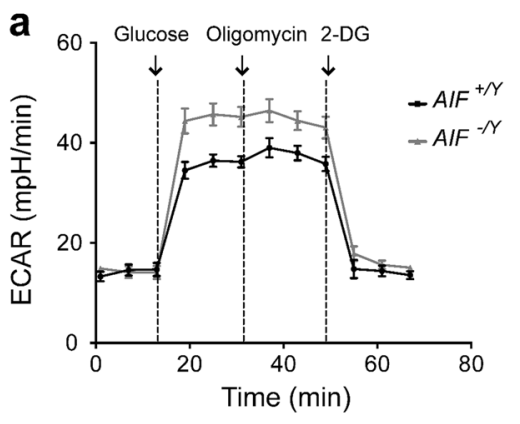

b

C

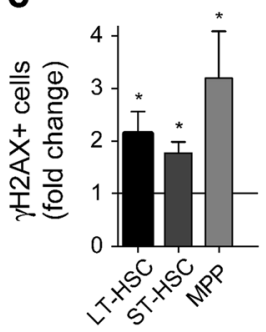

d
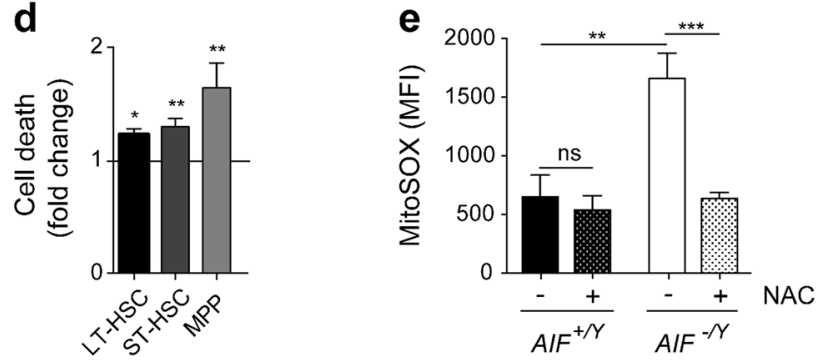

f
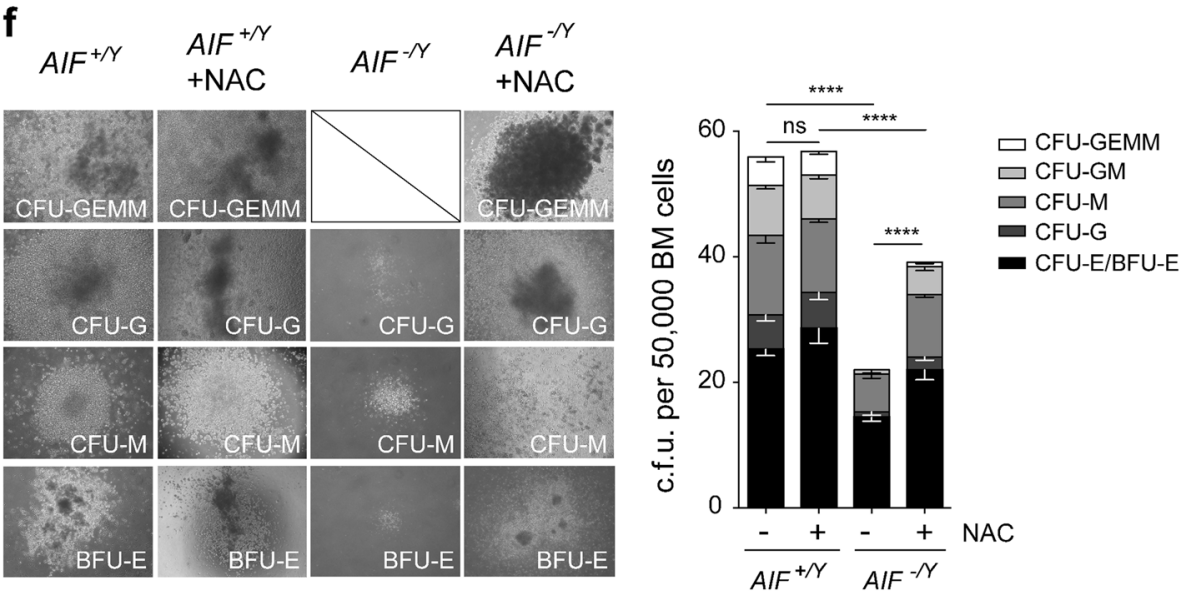

g

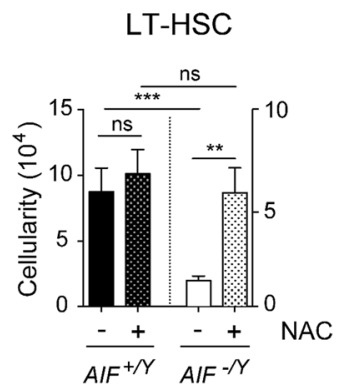

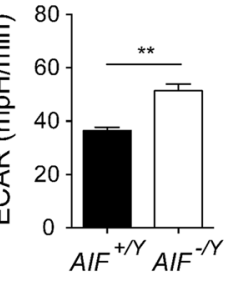

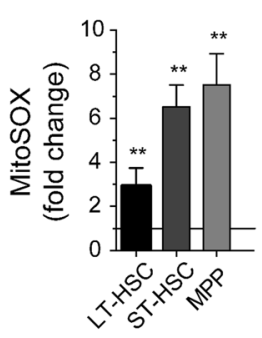

(n)


a

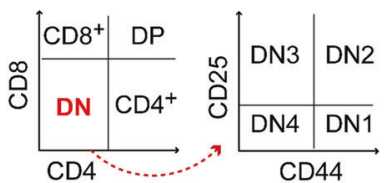

b

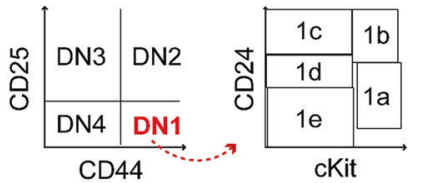

E15.5

E17.5
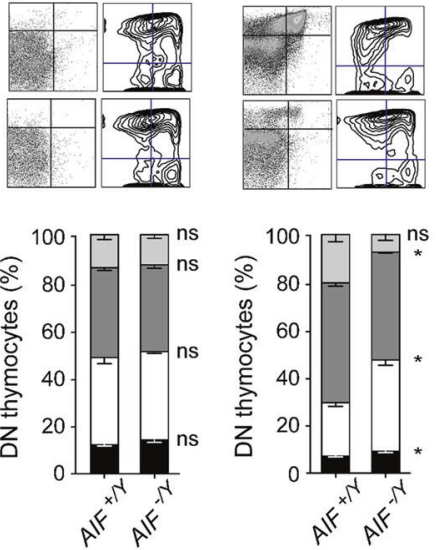

7 days
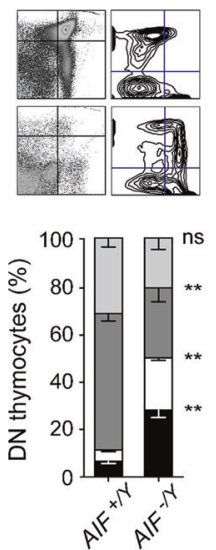

21 days
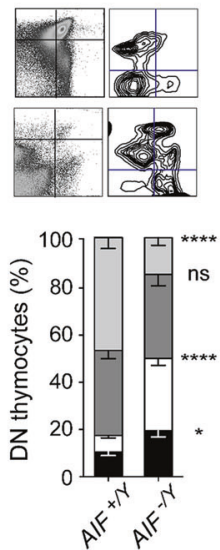

28 days
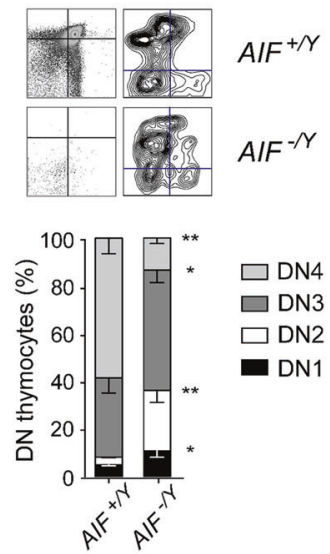

21 days

C

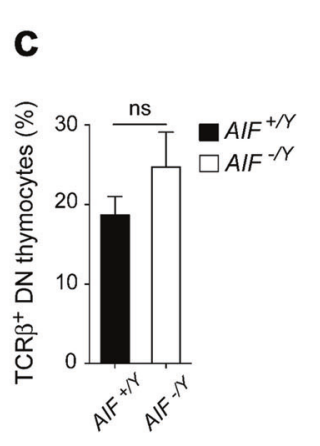

Fig. 5 Mitochondrial AIF loss led to the blockade of thymopoiesis at a DN immature state. a Left, Gating strategy. Right, Cytometric identification of DN1-DN4 subsets of $A I F^{+/ Y}$ and $A I F^{-/ Y}$ embryos (E15.5 and E17.5) and 7-, 21-, and 28-day-old neonates ( $n=7$ embryos/mice per group). b Left, Gating strategy. Flow cytometry assessment of the

their metabolism towards anaerobic glycolysis and FAO, respectively (Fig. 8).

In addition to its role in cell death [15-18, 20, 41], AIF is indispensable for OXPHOS function and mitochondrial energy production [11-13, 42-44]. According to the model of supercomplex organization [31,33], alterations on the ETC architectural structure yield OXPHOS dysregulation, along with respiratory defects, a reduced capacity for ATP production, and altered ROS generation; the same defects could be observed in $A I F^{-/ Y} \mathrm{BM}$ cells and thymocytes. Of note, AIF deficiency does not yield fully defective mitochondria. Indeed, this mitochondrial functionality appears critical for maintaining hematopoietic cell viability in neonatal mice and for yielding adaptive mitochondrial metabolic responses.

The role of AIF in the immune system was initially assessed in the $\mathrm{Hq}$ strain, which is not an AIF knockout mice $[26,27]$. In addition, the mitochondrial role of AIF in the T- and B-cell lineages was analyzed more recently by using Lck-Cre and CD19-Cre mouse models [28, 45]. A comparison between the immune alterations reported in these works and those characterizing the $A I F^{-/ Y}$ model

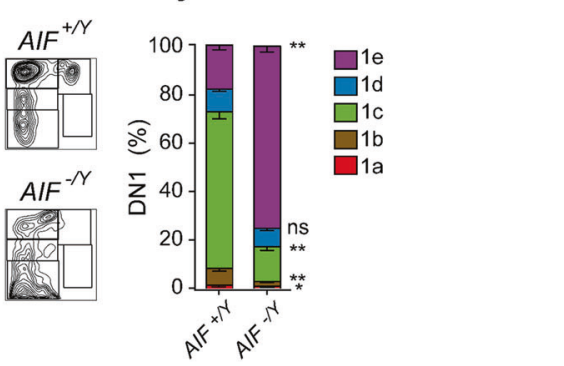

DN1 prothymocyte compartment from 7- and 21-day-old $A I F^{+/ Y}$ and $A I F^{-I Y}$ mice ( $n=6$ animals per group). c Frequency TCR $\beta$ chain positive DN thymocytes measured in $A I F^{+/ Y}$ and $A I F^{-/ Y}$ 21-day-old animals ( $n=8$ mice per group). Statistical significance was calculated by Mann-Whitney test. Bars represent mean \pm SEM

presented here indicates that the generation of bona fide hematopoietic AIF KO mice reveals a greater role for AIF in hematopoiesis. Specifically, thymocytes from $\mathrm{Hq}$ mice exhibited higher ROS levels, which appeared to be responsible for delayed DN3 development. By contrast, the T-cell developmental defects in our $A I F^{-/ Y}$ mice were rather strong: DP cell generation was not delayed but, instead, was fully compromised. This could be observed in fetuses, thus very early during thymic development. The analysis of the earliest thymic T-lineage precursors [46] reveals that their progressive loss is a consequence of a $\mathrm{BM}$ perturbation. However, it seems that the $\mathrm{DN} 1 \mathrm{a} / \mathrm{DN} 1 \mathrm{~b}$ subsets of the $A I F$ ${ }^{-/ Y}$ mice are still able to generate the DN2 population [46] that is always present in the thymus of the animals. Therefore, we could hypothesize that the delayed DN2/DN3 and DN3/DN4 transitions are independent from the canonical $\mathrm{T}$ precursors loss and, consequently, are associated to the OXPHOS/metabolic defaults caused by the AIF deficiency. Overall, our results seem to indicate that the developmental defaults characterizing the $A I F^{-I Y}$ thymocytes are cell intrinsic. The feeding of $A I F^{-/ Y}$ dams with an HFD allows for the generation of DP thymocytes without 
Fig. 6 AIF deficiency induced mitochondrial alterations and a ATP levels in thymocytes. a

Representative electron micrographs of $A I F^{+/ Y}$ and $A I F$

${ }_{-I Y}$ thymocytes. The black

arrows mark the mitochondria.

Bar: $0.5 \mu \mathrm{m}$. b Left, OCR of $A I F$

$+/ Y$ and $A I F^{-/ Y}$ thymocytes under basal conditions (initial rates) and in response to sequential treatment with Oligomycin,

FCCP, and Rotenone/Antimycin A. Arrows indicate the time of the addition of each reagent.

Right, Basal and maximal OCR of thymocytes expressed as a histogram ( $n=3$ independent experiments). c $\Delta \Psi \mathrm{m}$ assessment in $A I F^{+/ Y}$ and $A I F^{-/ Y}$ 21-day-old animals ( $n=18$ mice ROS levels measured in thymocytes from $A I F^{+/ Y}$ and $A I F$ ${ }^{-Y}$ 21-day-old animals $(n=18$ mice per group). $\mathbf{e}$

Mitochondrial mass of $A I F^{+/ Y}$ and $A I F^{-Y Y}$ thymocytes from 21 day-old animals $(n=18$ mice per group). f Glucose uptake measured by the assimilation of the D-glucose analog 2-NBDG in $A I F^{+/ Y}$ and $A I F^{-/ Y}$ thymocytes from 21-day-old mice $(n=5$ mice per group). g ATP levels recorded in $A I F^{+Y}$ and $A I F^{-Y Y}$ thymocytes from 21-day-old animals $(n=20$ mice per group). Statistical significance was calculated by

Mann-Whitney test. Bars

represent mean \pm SEM significant drop in the cellular per group). d Mitochondrial

a

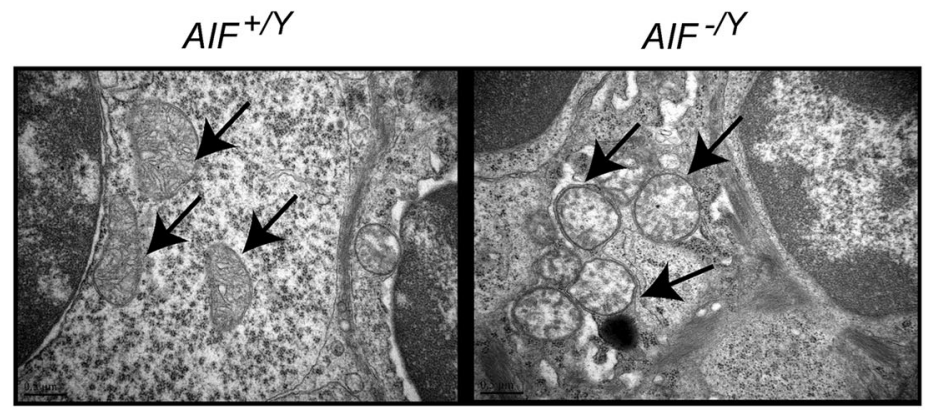

b
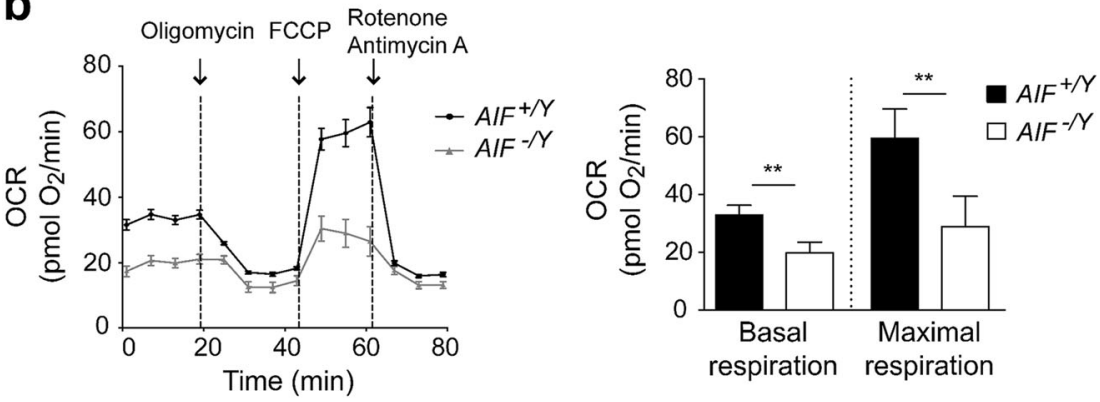

C
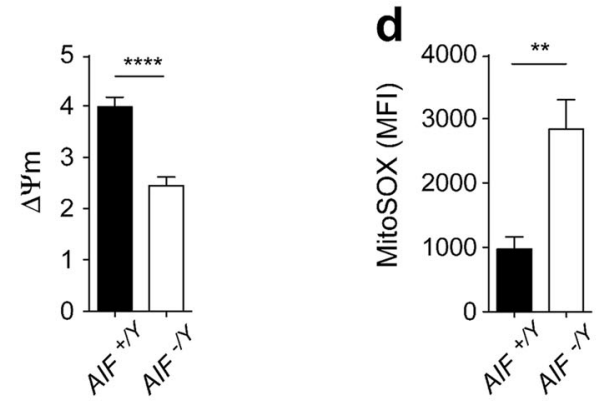

e
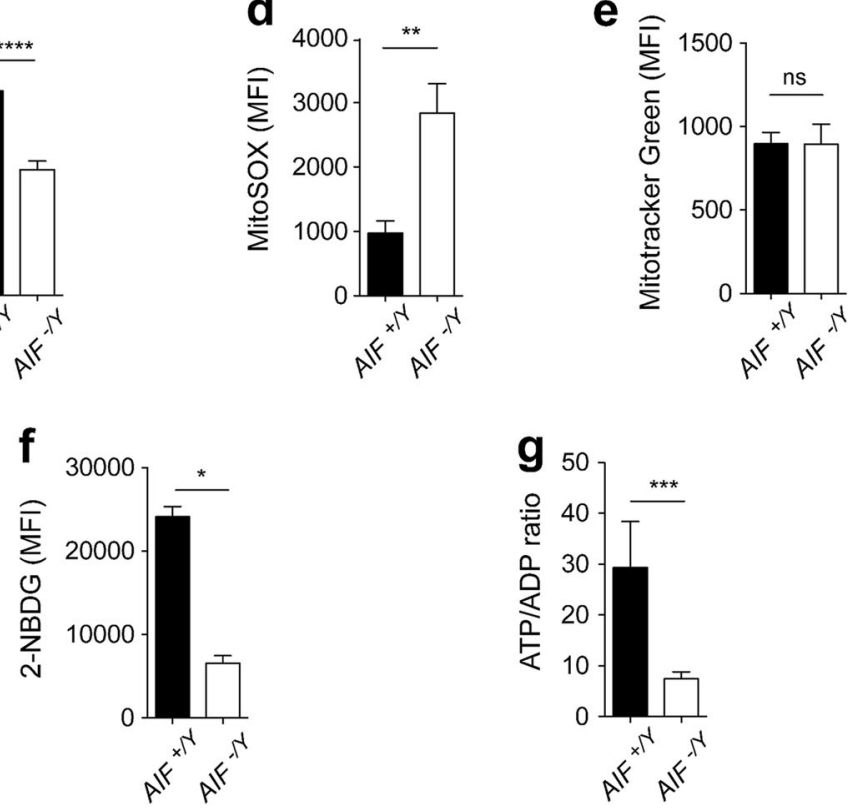

provoking a rescue in the HSC and progenitors (Supplementary Figure 10). Thus, it seems that the change of energy source is enough for the existing thymocytes to progress towards a DP phenotype.

In contrast to our results (and the results reported in the Hq strain) [26, 27], the immune alterations measured in the Lck-Cre model indicate that Aif ablation is associated with a loss in the number of peripheral $\mathrm{T}$ cells without defects in thymocyte development [28, 45]. This disagreement can be explained by the earlier elimination of AIF in our mice model compared to the latest Aif ablation in the Lck-Cre (around the DN3 stage). Indeed, the blockade at the DN stage observed in our $A I F^{-/ Y}$ mice, with alterations in the DN1, DN2, and DN3 stages, cannot be detected in the $L c k$-Cre animals. Of note, the adjustment in the mitochondrial metabolism profoundly alters DN1to-DN4 thymocyte development [9, 10]. Remarkably, in contrast to previous results on $\mathrm{Hq}$ and CD19-Cre AIF KO mouse models [26-28], our findings originally indicated that the loss of AIF undoubtedly affected B-cell and erythrocyte development. Here, the significant loss of T-cells observed in our mice model could also be implicated in the delayed B-cell development, as reported in other mice models [47]. 
a

b

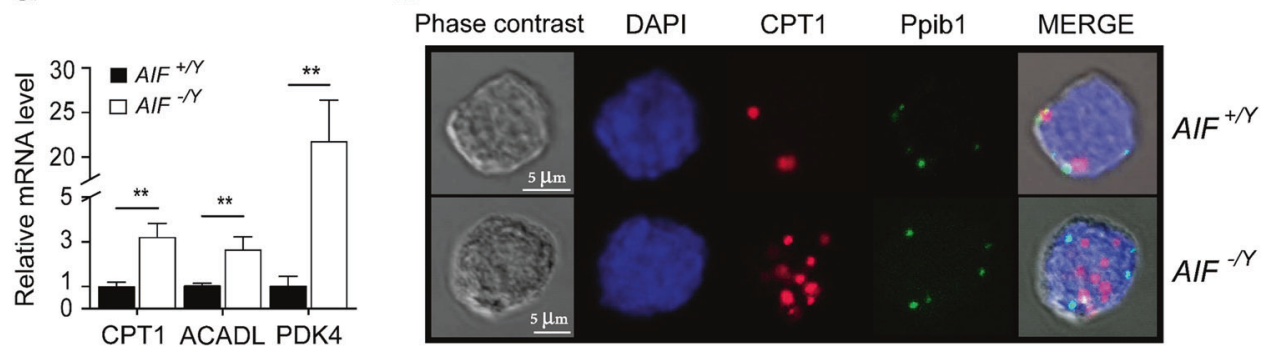

C
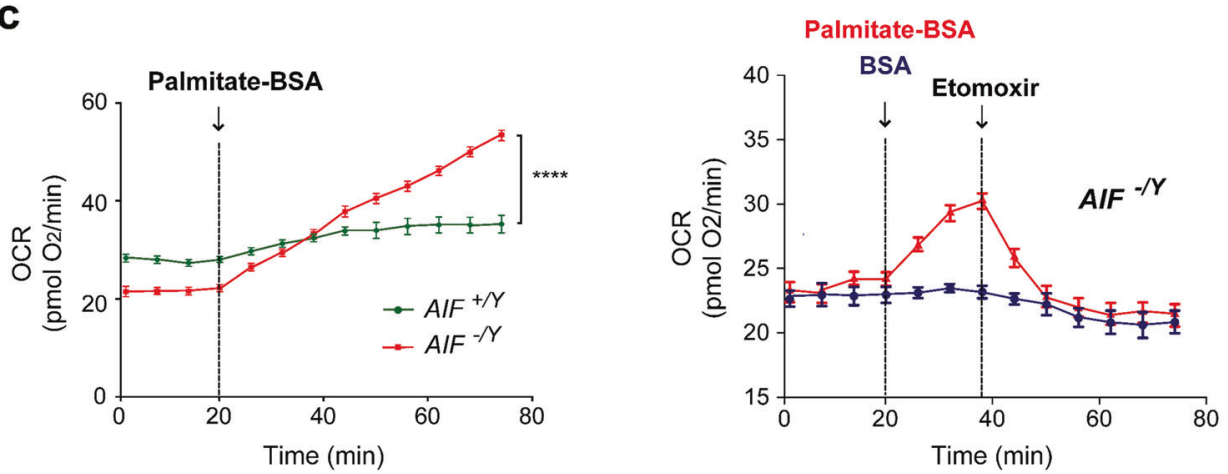

d
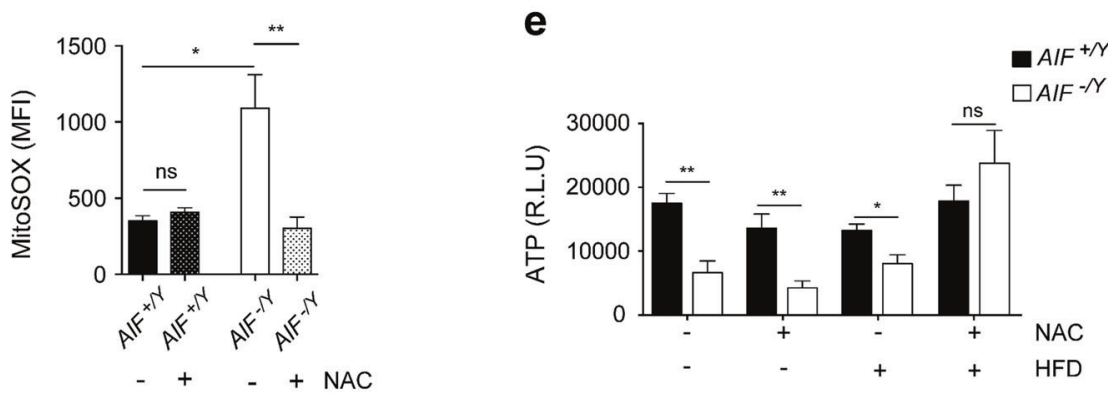

f
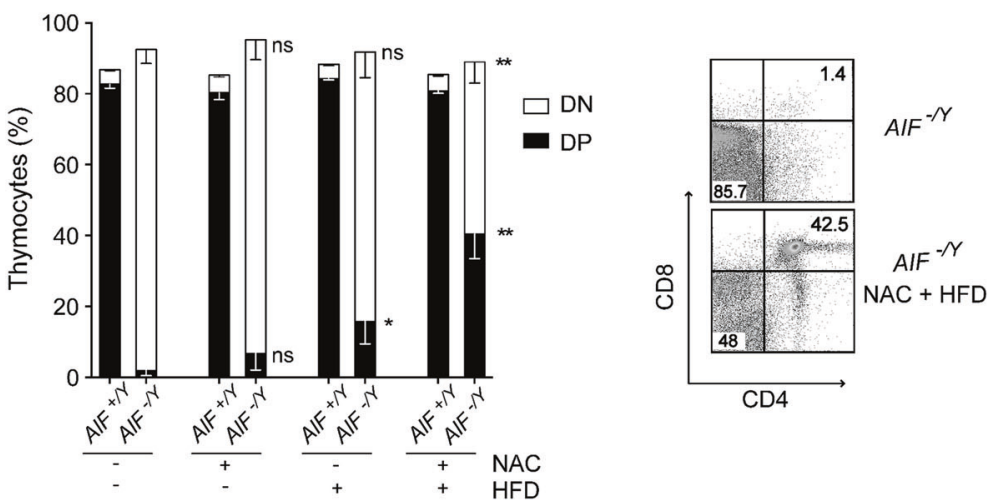

AIF deficiency leads to impaired survival of HSC, resulting in exhaustion of their pool, a reduced capacity to differentiate into colonies in methylcellulose assays, as well as an absence of cell-autonomous HSC repopulating potential in vivo. All these features appear to be a consequence of the high levels of ROS associated to the loss of AIF. ROS are toxic products of oxidative metabolism, which are necessary for intracellular signal transduction but can impair cellular functions when present in excess [48, 49]. An efficient way for HSC and progenitor cells to limit the excess of ROS is to favor anaerobic glycolysis over OXPHOS [1, 6]. Accordingly, we observed an increased use of glycolysis in $A I F^{-/ Y} \mathrm{BM}$ cells. In the presence of NAC, this metabolic shift seemed to be crucial for the rescue observed in both in vitro and in vivo experiments. Indeed, BM cells seemed to be exclusively 
4 Fig. 7 Mitochondrial OXPHOS breakdown favored fatty acid metabolism reprogramming in thymocytes. a CPT1, ACADL, and PDK4 mRNA levels determined by qPCR in $A I F^{+/ Y}$ and $A I F^{-Y Y}$ thymocytes from 21-day-old animals ( $n=10$ mice per group). The $18 \mathrm{~S}$ mRNA expression was used to normalize the data. b RNAscope fluorescent detection of Cpt $1 \alpha$ expression in representative $A I F^{+/ Y}$ and $A I F^{-/ Y}$ thymocytes from 21 -day-old animals. Cpt1 mRNA transcripts were stained with a specific probe conjugated to Atto 550 (red) and nuclei were counterstained with DAPI (blue). Ppib expression, detected with a specific probe conjugated to Alexa 488 (green), was used as a control. Phase contrast analysis was used to visualize cells. Compared to the similar expression of Ppib in $A I F^{+/ Y}$ and $A I F^{-/ Y}$ thymocytes, CPT1 was overexpressed in $A I F^{-/ Y}$ cells $(n=3$ independent experiments with similar results). c Left, OCR was measured in thymocytes from $A I F^{+/ Y}$ and $A I F^{-/ Y} 21$-day-old animals. After measurement of basal OCR, the FAO substrate Palmitate-BSA $(17 \mu \mathrm{M})$ (Agilent Technologies) was injected and OCR was assessed during 60 min. Arrows indicate the time of Palmitate-BSA addition. Right, OCR was measured in thymocytes from AIF ${ }^{-/ Y} 21$-day-old animals. After measurement of basal OCR, BSA control (blue line) or Palmitate-BSA FAO Substrate $(17 \mu \mathrm{M})$ (red line) were injected followed by $200 \mu \mathrm{M}$ Etomoxir addition to inhibit FAO. Arrows indicate the time of the injection of each reagent. OCR experiments were repeated three times with similar results. d Mitochondrial ROS levels measured in $A I F^{+/ Y}$ and $A I F^{-Y Y}$ thymocytes from 21-day-old newborns from dams supplied or not with NAC in drinking water ( $n=9$ animals per group). e Total ATP levels of $A I F^{+Y Y}$ and $A I F^{-/ Y}$ thymocytes from 21-day-old newborns from dams fed with standard control diet or high-fat diet (HFD) and supplied or not with NAC in drinking water ( $n=9$ animals per group). f Left, percentage of CD4 ${ }^{-} / \mathrm{CD}^{-}$ (DN) and $\mathrm{CD}^{+} / \mathrm{CD}^{+}(\mathrm{DP})$ thymocytes in 21-day-old $A I F^{+Y Y}$ and $A I F^{-/ Y}$ newborns from dams fed as in $\mathbf{e}(n=9$ animals per group). The statistical analysis refers to the comparison between the percentage of DN or DP thymocytes recorded in the $A I F^{-I Y}$ newborns from dams fed in standard conditions and the percentage of DN or DP thymocytes recorded in $A I F^{-Y Y}$ newborns from dams supplied either with NAC, HDF, or both. Right, representative flow cytometry panels of a CD4/CD8 labeling in $A I F^{-I Y}$ thymocytes from mice animals fed with a standard control diet $\left(A I F^{-/ Y}\right)$ or with an HFD supplied with NAC $\left(A I F^{-/ Y}\right.$ NAC + HFD). Statistical significance was calculated by Mann-Whitney test. Bars represent mean \pm SEM

adapted to a carbon metabolism and, contrary to thymocytes, appeared unable to shift towards FAO (Supplementary Figure 10a). The rescue of the HSC described in Fig. $4 \mathrm{~g}$ failed when changing the energy source from carbohydrates to fatty acids, a reaction observed even in the NAC presence (Supplementary Figure 10b). These results are in line with previous publications that, in similar feeding conditions, demonstrate that HFD causes decreased proliferation in LSK cells and a general suppression of progenitor cell populations in the BM [50].

ROS scavenging is also beneficial for T-cell rescue in $A I F^{-/ Y}$ mice, corroborating the previously reported detrimental role of uncontrolled mitochondrial ROS in thymocytes [51, 52]. However, the increased levels of ROS appear to be only partially responsible for the failure of $A I F^{-Y}$ thymocyte development. The developmental blockade characterizing these thymocytes also seems related to the lack of energy generated by these cells in a carbon environment. Surprisingly, the $A I F^{-Y}$ thymocytes could reprogram their metabolism by enhancing their capacity to assimilate fatty acids. As a result, the change of energy source from carbohydrates to fatty acids, combined with protection against the excess of ROS, significantly restores thymocyte development in $A I F^{-Y}$ animals. These results confirm that, as reported in other models with ETC alterations, it is possible to generate ATP by FAO in OXPHOS "damaged" mitochondria [53]. In future studies, it would appear necessary to analyze whether other pathways implicated in thymocyte metabolism reprogramming also have a role in our mouse strain (e.g., Akt activation has been linked to a blockade in the DN3-to-DN4 cell transition [54] and deletion of LKB1 results in accumulation of DN3 thymocytes and reduction in the number of thymocytes [55-57]).

Altogether, we have identified AIF as a new player that acts by fine-tuning the mitochondrial OXPHOS/metabolism in order to dictate the differentiation and fate of hematopoietic cells. We also revealed, in BM cells and thymocytes, the specific metabolic reprogramming associated to the dysfunction of the mitochondrial OXPHOS. It would be of interest to know whether similar phenomena occur in the immune cell compartments of patients suffering from mitochondriopathies. In any case, from a medical perspective, our data reveal potential therapeutic targets to regulate the immune responses in the context of autoimmunity, inflammation, or anti-tumor responses (e.g., by disrupting the mitochondrial metabolism).

\section{Materials and methods}

\section{Mice}

All mice were housed at the Cordeliers Center animal facility under strictly controlled, specific pathogen-free conditions (agreement B75-06-12). Experiments were performed in accordance with ARRIVE ethical guidelines and with the approval of the French Ministry of Agriculture (agreement 1675). Mice were maintained with a rodent diet (R03, Scientific Animal Food \& Engineering Diets) and water was made available ad libitum in a vivarium with a 12-h light-dark cycle at $22^{\circ} \mathrm{C}$. In specific experiments, dams were fed on a standard control diet (CD; D12450J; Research Diets) or a high-fat diet (HFD; D12492i; Research Diets) before mating and kept on the same diet during pregnancy and lactation.

To generate the hematopoietic cell-specific $A I F^{-/ Y}$ mice, the exon 11 of Aifml (Pdcd8, Aif) was flanked by LoxP sites using standard gene-targeting techniques (Supplementary Figure 1). After at least 15 episodes of interbreeding in the C57BL/6 J background, the Aifm $1^{f /+}$ females were crossed with C57BL/6 J Vav1-Cre males (Jackson Laboratory) to obtain the $A I F^{-/ Y}$ mice (Aifm $I^{f / Y}$; Vavl-Cre ${ }^{+}$). The $A I F^{+/ Y}$ littermates were used as control WT mice (defined as $A I F^{+/ Y}$ 
Fig. 8 Schematic representation of the consequences of the early Aif ablation in the

hematopoietic cells. The loss of AIF in the hematopoietic cells resulted in a defective mitochondrial OXPHOS that was characterized by $\Delta \Psi \mathrm{m}$ loss, high ROS generation, and drop in ATP levels. These alterations provoke pleiotropic consequences in neonatal and adult $A I F^{-/ Y}$ mice, including a progressive pancytopenia and $\mathrm{T}$ cell, B-cell, and erythroid developmental defaults. BM cells and thymocytes utilize different adaptive metabolic mechanisms: BM cells counterbalance the AIFmediated OXPHOS dysfunction by the stimulation of mitochondrial biogenesis and a shift towards anaerobic glycolysis, whereas thymocytes favor the fatty acid metabolism
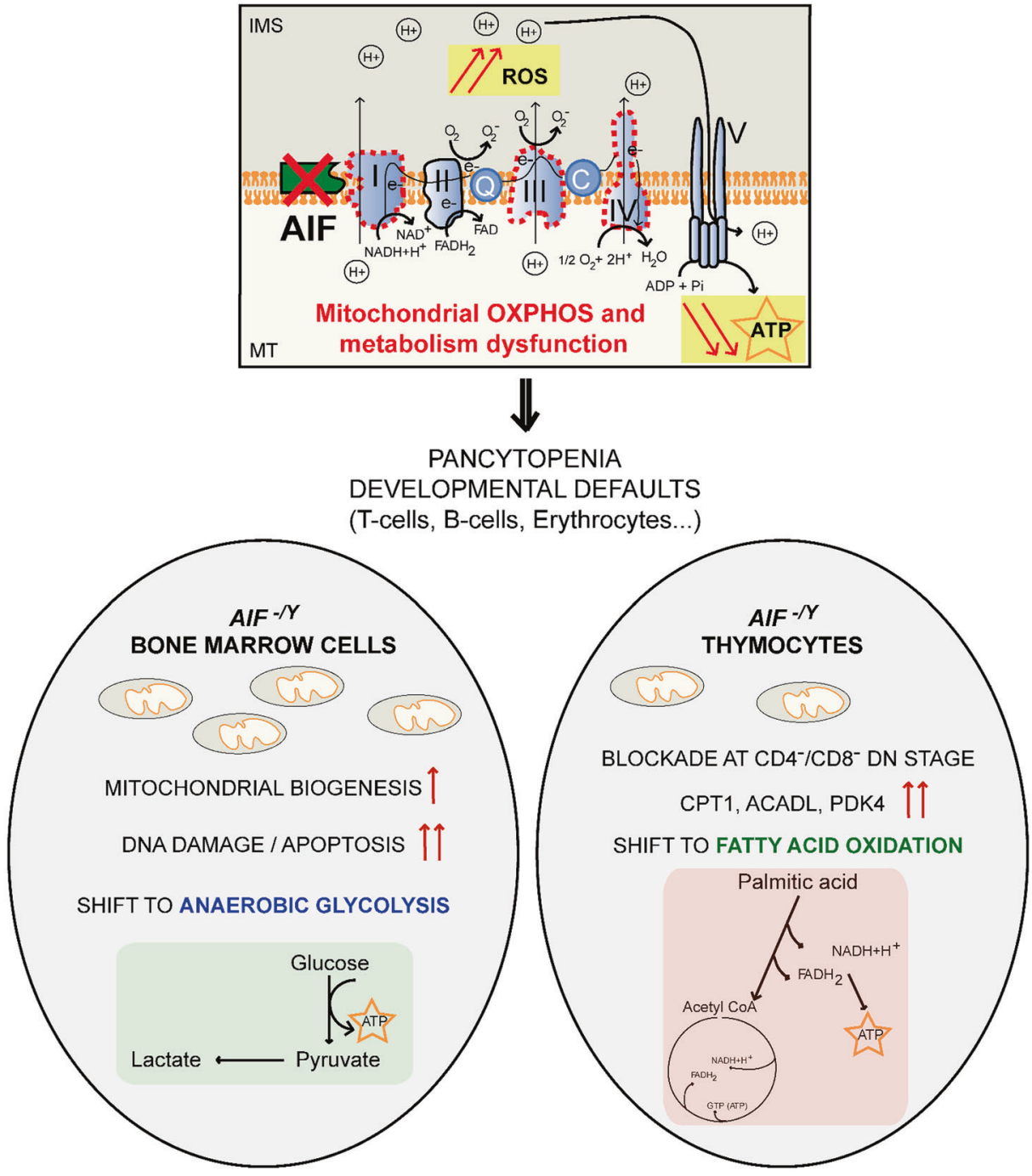

in the manuscript). No significant differences were observed

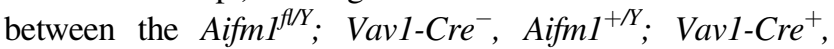

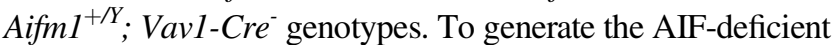
mice in adult animals, the Aifm $1^{f /+}$ females were crossed to Rosa26-CreERT2 males (provided by Dr Anton Bernes, NCI, Amsterdam, The Netherlands) [30] and, 3 months after birth, the Aifm $I^{f / Y}$; Rosa26-CreERT2 ${ }^{+}$animals were injected daily with tamoxifen (five intra-peritoneal injections; $50 \mathrm{mg} /$ $\mathrm{kg}$ body weight). The Aifm ${ }^{f / Y}$; Rosa26-CreERT2 ${ }^{-}$ tamoxifen-injected mice were used as control (WT) animals. No significant differences were observed with the other nonAIF deleted genotypes generated by the crossing.

To perform the antioxidant treatment of the lactating mice, N-acetyl-L-cysteine NAC (Sigma) was administered to the dams in drinking water at $2 \mathrm{~g} / \mathrm{kg}$ of body weight per day (starting 3 days after giving birth and until newborn sacrifice). Mice peripheral blood samples were collected in EDTA-coated tubes and cell counts were analyzed in an automated counter (Scil Vet abc; SCIL GmbH).

\section{Flow cytometry}

For the different immunophenotyping approaches, the cellular suspensions from thymi, spleens, and BM were labeled with the corresponding antibody in FACS Buffer (2 mM EDTA 2\% FCS PBS), recorded in a FACSCanto II (BD Biosciences), and analyzed using the FlowJo software (TreeStar Inc). All flow cytometry samples were treated with FcBlock (2.4G2; BD Biosciences) prior to their incubation with primary antibodies, except when identifying the BM HSC subsets.

The following antibodies (clone) were used: CD44 (IM7), CD45R/B220 (RA3-6B2), CD34 (RAM34), CD127 (A7R34), CD3e-PE (145-2C11), Gr-1 (RB6-8C5), CD69 (H1-2F3), Foxp3 (FJK-16s), CD135 (Flt3) (A2F10), CD45.1 (A20), CD150 (SLAM) (TC15-12F12.2), CD24 (M1/69), $\gamma \delta$ T cells (GL3), CD4 (RM4-5), Ter119 (Ter119), F4/80 (BM8), streptavidin, Sca-1 (D7), TCR-bchain, TC (GL3), CD11c (N418), CD62L (MEL-14), IgM (R6-60.2), CD71 (RI7217), CD34 (RAM34), CD4 (RM4- 
5), CD19 (ebio1D3), CD8 $\alpha$ (53-6.7), NK-1.1 (PK136), IgD (11-26 c.2a), CD11b (M1/70), CD25 (PC61), CD117/cKit (2B8), CD16/32 (HM48- 1 ). Antibodies were obtained from BioLegend, BD Biosciences, or eBioscience.

For detection of LT-HSC, ST-HSC, MPP, MEP, CMP, and GMP cells, BM cells were incubated with a cocktail of biotinylated antibodies against Mac-1 $\alpha$ (CD11b), Gr-1 (Ly6G/C), Ter119, CD3, CD4, CD8a, and B220 (BD Biosciences) followed by detection with streptavidin conjugated to PerCP-Cy5.5. Then, cKit, Sca-1, CD34, CD135, and CD16/32 fluorochrome-conjugated antibodies were added to the mix. To define CLP cells and following a similar approach, cKit, Sca1, and CD127 fluorochrome-conjugated antibodies were added to the initial cocktail. For congenic strain discrimination, anti-CD45.1 and anti-CD45.2 antibodies (BD Biosciences) were used.

For the analysis of the DN compartment, thymocytes were incubated with a cocktail of biotinylated antibodies against CD8a, Mac-1 $\alpha$ (CD11b), Gr-1 (Ly-6G/C), Ter119, CD3, and B220 (BD Biosciences) followed by detection with streptavidin conjugated to PerCP-Cy5.5. Then, CD24, CD25, CD44, and cKit antibodies were added to the mix. DN1 subpopulations were defined as: DN1a (cKit+CD24 low), DN1b (cKit+CD24+), DN1c (cKit-CD24+), DN1d (cKit-CD24 low), and DN1e (cKit-CD24-).

Isolation of $\mathrm{DN}$ fraction was performed by negative selection. In brief, thymocytes were pre-incubated with a mix of CD4 and CD8 magnetically labeled antibodies (BD Biosciences) and then placed on the BD IMag Cell Separation System, following the manufacturer's instructions.

\section{Mitochondrial and cellular analysis}

For mitochondrial ROS, mitochondrial mass, and $\Delta \psi \mathrm{m}$ measurements, cells were incubated with MitoSOX (5 $\mu \mathrm{M})$, Mitotracker Green and Mitotracker Red (100 nM each) (Invitrogen), respectively, before assessment in a FACSCanto II.

For analysis of glucose uptake, BM cells and thymocytes were incubated $\left(30 \mathrm{~min} ; 37^{\circ} \mathrm{C}\right)$ in glucose-free DMEM with 2-NBDG $(100 \mu \mathrm{M}$; Invitrogen $)$ prior to flow cytometry assessment.

For DNA damage detection, cells were first labeled for HSC markers, incubated with a cytofix-cytoperm buffer (20 min on ice), and washed twice in Permwash buffer (BD Biosciences). Cells were then incubated with anti- $\gamma \mathrm{H} 2 \mathrm{AX}$ antibody (16-202 A; Millipore) and analyzed in a FACSCanto II.

Apoptosis (cell death) was assessed by flow cytometry using Annexin-V-APC $(0.1 \mu \mathrm{g} / \mathrm{ml}$; BD Biosciences $)$ and 7AAD double labeling ( $1 \mu \mathrm{g} / \mathrm{ml}$; BD Biosciences). In some experiments, BM cells and thymocytes were pretreated during $30 \mathrm{~min}$ with the broad-spectrum caspase inhibitor QVD-OPh (QVD, $1 \mu \mathrm{M})$ before performing metabolic and cytometric tests.
ATP was analyzed in $10^{4} \mathrm{BM}$ cells or thymocytes with an ATP bioluminescent kit (Abcam) in an Infinite M100 PRO plate reader (Tecan) and expressed as total ATP or ATP/ADP ratio.

For lactate measurement, cells were cultured for $2 \mathrm{~h}$ in a serum-free medium and centrifuged. Then, the supernatant was used to quantify extracellular L-lactate with a kit from Cayman Chemical Company in an Infinite M100 PRO plate reader (Tecan).

\section{Metabolomic approach}

The whole metabolomic assay was performed on $3 \times 10^{7}$ BM cells by GC-TOF-MS on a LECO Pegasus III with an Agilent 6890 N GC system (Massy, France) and an Agilent 7683 automatic liquid sampler as described [58]. The column used was an RTX-5 w/integra-Guard (30 m, $0.25 \mathrm{~mm}$ internal diameter $+10 \mathrm{~m}$ integrated guard column) (Restek, Evry, France). Metabolites were extracted from samples using a methanol/water mixture $(80 / 20 \mathrm{v} / \mathrm{v})$. Peak identity was established by comparison of the fragmentation pattern with MS available databases, using a match cut-off criterion of 700/1000 and by retention time using the alkane series as retention standards. The integration of peaks was performed using the LECO Pegasus software (Garges-lès-Gonesse, France). The relative amount of each metabolite was calculated on the basis of the corresponding peak area compared with an internal standard (Ribitol).

\section{Clark electrode}

OCR were measured in $5 \times 10^{7} \mathrm{BM}$ cells as previously described [59]. Respiration was measured in nonpermeabilized cells under basal conditions and in response to the sequential addition of oligomycin $(10 \mu \mathrm{M})$, fluorocarbonyl cyanide phenylhydrazone (FCCP; $5 \mu \mathrm{M}$ ), amytal $(2 \mathrm{mM})$, and azide $(5 \mathrm{mM})$.

\section{Measurement of OCR, ECAR, FAO, and glutamine dependency by Seahorse}

Bone marrow cells or thymocytes isolated from $A I F^{+/ Y}$ and $A I F^{-/ Y}$ animals were seeded at a density of 400,000 to 800,000 cells per well in an XFe96 cell culture microplate pretreated with CellTak (Corning). Cells were balanced for 1 $\mathrm{h}$ in unbuffered XF assay media (Agilent Technologies) supplemented for OCR analysis with either $2 \mathrm{mM}$ Glutamine, $10 \mathrm{mM}$ Glucose and $1 \mathrm{mM}$ Sodium Pyruvate or just $2 \mathrm{mM}$ Glutamine for ECAR measurement. For OCR measurements, compounds were injected during the assay at the following final concentrations: Oligomycin (ATP synthase inhibitor, 1 $\mu \mathrm{M}$ ), FCCP (uncoupling agent measuring the maximal respiration capacity; $1 \mu \mathrm{M}$ ), Rotenone and Antimycin A 
(ETC inhibitors; $1 \mu \mathrm{M}$ ). For ECAR measurements, Glucose $(10 \mathrm{mM})$, Oligomycin $(1 \mu \mathrm{M})$, and 2-Deoxyglucose (2-DG, glycolytic inhibitor; $500 \mathrm{mM}$ ) were injected.

The activities of complex I, II/III and IV were measured in BM cells plated in mitochondria assay buffer $(660 \mathrm{mM}$ Mannitol, $210 \mathrm{mM}$ Sucrose, $30 \mathrm{mM}$ KH2PO4, $15 \mathrm{mM}$ $\mathrm{MgCl} 2,6 \mathrm{mM}$ HEPES, $3 \mathrm{mM}$ EGTA, $0.6 \%$ fatty acid-free BSA) supplemented with $4 \mathrm{mM}$ ADP and $1 \mathrm{nM}$ XF Plasma Membrane Permeabilizer Reagent (XF PMP, Agilent Technologies). The complex I substrates (1 mM Malate and $10 \mathrm{mM}$ Pyruvate), the complex II substrate (10 mM Succinate) or the complex IV substrates $(100 \mu \mathrm{M}$ TMPD and 10 $\mathrm{mM}$ Ascorbate) were then added and OCR determined.

For FAO tests, cells were plated in FAO assay media (110 mM NaCl, $4.7 \mathrm{mM} \mathrm{KCl}, 1.25 \mathrm{mM} \mathrm{CaCl}, 2 \mathrm{mM}$ $\mathrm{MgSO}_{4}, 1.2 \mathrm{mM} \mathrm{NaH} \mathrm{PO}_{4}$ ) supplemented with $2.5 \mathrm{mM}$ Glucose, $0.5 \mathrm{mM}$ Carnitine, and $5 \mathrm{mM}$ HEPES. After measurements of basal OCR, BSA control $(100 \mu \mathrm{M})$, Palmitate-BSA FAO Substrate $(17 \mu \mathrm{M})$ (Agilent Technologies), or Etomoxir $(200 \mu \mathrm{M})$ were injected.

For the glutamine dependent assessment, BM cells were plated in unbuffered XF assay media (Agilent Technologies). Then, in ECAR measurements, Glucose (10 mM), Glutamine $(2 \mathrm{mM})$, or Glucose+Glutamine $(10 \mathrm{mM}$ and 2 $\mathrm{mM}$, respectively) were injected followed by 2-DG (500 $\mathrm{mM})$ inhibition. In the OCR assessment, Glutamine (2 $\mathrm{mM})$ and BPTES $(3 \mu \mathrm{M})$ were sequentially injected.

\section{High-resolution fluorescent in situ hybridization by RNAscope (FISH by RNAscope)}

RNAscope assessment was strictly performed following manufacturing procedures (Advanced Cell Diagnostics, inc.; ACD) [34]. In brief, thymocytes purified from $A I F^{+/ Y}$ and $A I F^{-/ Y}$ animals were fixed with $4 \%$ paraformaldehyde for 30 min at $4{ }^{\circ} \mathrm{C}$, washed with PBS pH 7.2, centrifuged (Cytospin 3, Shandon) at $700 \mathrm{rpm}$ for $3 \mathrm{~min}$ on Superfrost Plus slides, dehydrated and permeabilized by Protease III digestion (ACD) for $10 \mathrm{~min}$ at RT. Then, slides were hybridized with mouse Cpt1 (conjugated to Atto 550) and Peptidylprolyl Isomerase B (Ppib, conjugated to Alexa 488; mRNA control) probes. Nuclei were visualized using DAPI staining. Hybridization of a probe against the Bacillus subtilis dihydrodipicolinate reductase (DapB) gene was used as a negative control. The resulting hybridized slides were imaged using a LSM 710 Zeiss confocal microscope and analyzed using the image J software. RNAscope experiments were repeated three times on different slide sets with similar results.

\section{Retroviral transduction in BM and FL cells}

Murine AIF cDNA was cloned into the MSCV-IRES-GFP retroviral vector. Viruses were generated into $293 \mathrm{~T}$ cells by X-tremeGene 9 (ROCHE) transient transfection of the retroviral constructs and the packaging plasmids EcoPack/Psi Eco. $48 \mathrm{~h}$ post transfection, retroviral supernatants were harvested, clarified by filtration, and kept at $-80^{\circ} \mathrm{C}$ until use. For transduction in colony-forming assays and $\mathrm{FL}$ in vivo transfer, $A I F^{+/ Y}$ or $A I F^{-/ Y}$ donor embryos were collected at E15.5 and FL cells were prepared and seeded. For transduction in colony-forming assays, BM $A I F^{+/ Y}$ or $A I F^{-/ Y}$ cells were obtained from 7day-old animals and prepared similarly to the FL cells. After an overnight culture in RPMI 1640 supplemented with $10 \%$ FBS, $10 \mathrm{ng} / \mathrm{ml} \mathrm{mIL-3,} 10 \mathrm{ng} / \mathrm{ml} \mathrm{mIL-6,} \mathrm{and} 10 \mathrm{ng} /$ $\mathrm{ml} \mathrm{mSCF}$, cells were spin-infected with either MSCV-IRESGFP or MSCV-mAIF-IRES-GFP viral supernatants. Then, cells were plated in M3434 methylcellulose medium or transferred in recipient mice CD45.1 as described below. Note that the percentage of cell transduction is similar in FL and BM cells $(\sim 5 \%)$.

\section{Transplantation assays}

CD45.1 C57B1/6 J mice were lethally irradiated and reconstituted by retro-orbital injections with BM, LSK, or FL cells from $A I F^{-/ Y}$ or $A I F^{+/ Y}$ CD45.2 animals collected between 3 and 10 days after mice birth (BM and LSK) or between E12.5 and E15.5 (FL cells). In non-competitive experiments, $1 \times 10^{6} \mathrm{BM}$ CD45.2 donor cells were injected in CD45.1 mice. In competitive assays, $1 \times 10^{4}$ CD45.2 donor LSK cells were mixed with $2.5 \times 10^{5}$ CD45.1 competitor BM cells before injection. In the rescue performed with FL cells transduced or not with the mAIF cDNA, $2 \times$ $10^{6} \mathrm{FL} \mathrm{CD} 45.2$ cells were mixed with $1 \times 10^{6} \mathrm{CD} 45.1 /$ CD45.2 BM cells before injection in CD45.1 mice. Engraftment in the immune organs in non-competitive and competitive transplantation assays with BM and LSK cells was assessed by CD45.2 donor contribution using FACS analysis of peripheral blood samples at 2 and 6 weeks post transplantation and at the moment of sacrifice (8 weeks) in the immune organs. In competitive experiments with FL transduced cells, samples were analyzed at 1 and 3 months post engraftment in the peripheral blood and at 4 months post engraftment in the BM. In each experiment, transplants were carried out with five donors per genotype and with, at least, five recipient mice per donor.

\section{CFU assays}

Whole FL cells or BM cells were plated in duplicate in MethoCult M3434 (StemCell Technologies) and cultured at $37^{\circ} \mathrm{C}$ in $5 \% \mathrm{CO} 2$ for 10 days before colony counting. Colonies were scored according to morphological criteria [60]. In selected experiments, NAC $(250 \mu \mathrm{M})$ was added to methylcellulose at the time of cell plating. 


\section{Electron microscopy}

BM cells or thymocytes were centrifuged $(10 \mathrm{~min} ; 300 \mathrm{~g})$ and the resulted pellets were fixed at $4{ }^{\circ} \mathrm{C}$ for $2 \mathrm{~h}$ in $2.5 \%$ glutaraldehyde in $0.1 \mathrm{M}$ phosphate buffer ( $\mathrm{pH} 7.3$ ), postfixed for $1 \mathrm{~h}$ in $1 \%$ buffered osmium tetroxide, dehydrated through a graded ethanol series, and embedded in Epon 812. The ultrathin sections were counterstained with $2 \%$ aqueous uranyl acetate for $30 \mathrm{~min}$, then with lead citrate for $10 \mathrm{~min}$ and finally viewed under a Philips $\times 100$ electron microscope.

\section{Immunoblotting}

Freshly isolated BM, spleen, thymus, and FL cells were lysed in buffer containing $50 \mathrm{mM}$ Tris $\mathrm{pH} 7.5,150 \mathrm{mM}$ $\mathrm{NaCl}, 1 \mathrm{mM}$ EDTA, $1 \%$ Triton X-100, and the protease/ phosphatase inhibitor cocktail from Roche. Protein concentration was determined using the BioRad Protein Assay. Equal amounts of total proteins $(50 \mu \mathrm{g})$ were loaded on linear SDS-PAGE gels and transferred onto a PVDF or nitrocellulose membrane. Membrane blocking and antibody incubations were performed in TBS $0.1 \%$ Tween 20 plus 5\% non-fat dry milk. The primary antibodies used were: AIF (D20, Santa Cruz), NDUFA9 (20C11B11B11, Life technologies), SDHA (2E3GC12FB2AE2, Life technologies), UQCRC2 (13G12AF12BB11, Abcam), COX4I2 (20E8C12, Abcam), ATP5B (3D5AB1, Life technologies), VDAC1 (ab15895, Abcam), and $\beta$-actin (AC15, Sigma). Immunoreactive proteins were detected using HRP-conjugated secondary antibodies and visualized with SuperSignal West Dura chemiluminescence kits (Thermo Scientific). Immunoblot images were acquired on a MF-ChemiBIS 4.2 (DNR Bio-Imaging Systems). AIF, NDUFA9, SDHA, UQCRC2, COX4I2, and ATP5B protein expression were quantified using the Multi Gauge 3.0 software (Fujifilm Life Sciences). The optical density was normalized according to an endogenous background and the OD mean of $A I F^{-/ Y}$ ETC proteins was expressed as a percentage relative to the OD mean obtained in the proteins from $A I F^{+/ Y}$ cells $(=100 \%)$.

\section{Quantitative RT-PCR}

Total RNA was extracted using the Nucleospin RNA II kit (Macherey-Nagel). cDNA was prepared using Superscript II reverse transcriptase (Life Technologies). Quantitative RTPCR was performed using the TaqMan Gene Expression Assays (Life Technologies) for Pgcla (Peroxisome proliferator-activated receptor gamma coactivator 1-alpha), Cptl (carnitine palmitoyltransferase I), Acadl (Acyl-CoA Dehydrogenase, Long Chain), or Pdk4 (pyruvate dehydrogenase kinase, isozyme 4). PCR reactions were performed in duplicate using TaqMan Fast advanced Master Mix (Life Technologies). The products were amplified in a
ViiA7 Real-time PCR System (Life Technologies) at $60{ }^{\circ} \mathrm{C}$ for 40 cycles. Data were analyzed using the comparative threshold cycle method. The housekeeping expression of $18 S$ was utilized to normalize the data.

\section{Histology}

Femurs were fixed in 4\% PFA and embedded in paraffin, and $4 \mu \mathrm{m}$ thick sections were stained with hematoxylin-eosin. Images were obtained using an Olympus AX70 microscope and a Sony XCD-U100CR color digital camera. The microscope was equipped with a $\times 10$ / 0.30 objective lens. All images were acquired using the Archimed software (Microvision Instrument) and analyzed using Adobe Photoshop 8.0.1 (Adobe Systems).

\section{Statistics}

Results, displayed as mean \pm SEM, were statistically analyzed using the GraphPad Prism software. Kaplan-Meier curves were produced and analyzed by log-rank tests. Statistically significant $p$-values were indicated as follows: * $p \leq 0.05, * * p \leq 0.01, * * * p \leq 0.001, * * * * p \leq 0.0001$. All the experiments were independently repeated at least three times. In the metabolomic assessment, the biostatistic analysis was performed with the Mev software (http://www. $\mathrm{tm} 4 . \mathrm{org} / \mathrm{mev} /$ ) and the statistical significance was calculated using Student's $t$-test. For the hierarchical clustering analysis, the data were normalized using the following formula:

$$
\begin{array}{r}
\text { Normalized value }=[(\text { Value })-\operatorname{Mean}(\text { Row })] / \\
{[\text { Standard deviation }(\text { Row })] .}
\end{array}
$$

Acknowledgements We thank G Delespesse, M Rubio, K Waahara, and N Baba (CRCHUM, Montréal, Canada) for support and helpful tips and discussions, Sophie Ezine and Victoria Michaels (INEM UMR-S1151, CNRS UMR 8253 Université Paris Descartes-Site Broussais, Paris, France) for invaluable help in thymocyte labeling and flow cytometry analysis, the animal facility staff (CEF Cordeliers, Paris, France) for mice housing support, Christophe Klein for help in confocal image acquisition (CICC, Cordeliers, Paris, France), V Della Valle and D Roos-Weil (U1170 INSERM, Villejuif, France) for advice in transplantation and colony-forming assays, A Prola and C Lemaire (UMRS 1180, Châtenay-Malabry, France) for help in the set-up of Clark electrode oxygen consumption assays and for providing amytal, and M Segade (Houston, US) for proofreading. This work was supported by Fondation ARC (PJA20151203407), Ligue Contre le Cancer-Comité de Paris (RS15/75-63 and RS16/75-50), Association Laurette Fugain (ALF 15/09), Fondation de France-Comité Cancer, Fondation pour la Recherche Medicale, French National Research Agency (ANR-09-BLAN-0247 and ANR-12-EMMA-0045), and French National Cancer Institute (INCa-5839) (to SA Susin). LC received $\mathrm{PhD}$ fellowship support from ENS-Cachan, Société Française d'Hématologie, and Fondation ARC, as well as travel grants from Boehringer Ingelheim and Journal of Cell Science. AB received PhD fellowships from the French Research Ministry and Société Française d'Hématologie. 
Author Contributions $\mathrm{LC}$ and $\mathrm{AB}$ designed experiments, performed in vitro and in vivo studies, interpreted the data and helped to write the manuscript. M-NB-N, LS, LD, LV, and MB, performed in vitro and/or in vivo experiments. IN designed and performed the Seahorse metabolic assessment. CL performed histological analyses. KG and DC carried out electron microscopy. FG, performed metabolomic approach. VQV, MS, $\mathrm{TM}$, and $\mathrm{OAB}$ provided reagents, analyzed data, and/or contributed to the experimental design and analysis. SAS supervised the study, designed experiments, interpreted the data, and wrote the manuscript.

\section{Compliance with ethical standards}

Conflict of interest The authors declare that they have no conflict of interest.

\section{References}

1. Folmes CD, Dzeja PP, Nelson TJ, Terzic A. Metabolic plasticity in stem cell homeostasis and differentiation. Cell Stem Cell 2012;11:596-606.

2. Zhang CC, Sadek HA. Hypoxia and metabolic properties of hematopoietic stem cells. Antioxid Redox Signal 2014;20: 1891-901.

3. Suda T, Takubo K, Semenza GL. Metabolic regulation of hematopoietic stem cells in the hypoxic niche. Cell Stem Cell 2011;9:298-310.

4. Maryanovich M, Zaltsman Y, Ruggiero A, Goldman A, Shachnai L, Zaidman SL, et al. An MTCH2 pathway repressing mitochondria metabolism regulates haematopoietic stem cell fate. Nat Commun 2015;6:7901.

5. Ito K, Suda T. Metabolic requirements for the maintenance of selfrenewing stem cells. Nat Rev Mol Cell Biol 2014;15:243-56.

6. Pearce EL, Pearce EJ. Metabolic pathways in immune cell activation and quiescence. Immunity 2013;38:633-43.

7. Arranz L, Urbano-Ispizua A, Mendez-Ferrer S. Mitochondria underlie different metabolism of hematopoietic stem and progenitor cells. Haematologica 2013;98:993-5.

8. Simsek T, Kocabas F, Zheng J, Deberardinis RJ, Mahmoud AI, Olson EN, et al. The distinct metabolic profile of hematopoietic stem cells reflects their location in a hypoxic niche. Cell Stem Cell 2010;7:380-90.

9. Chen H, Yang T, Zhu L, Zhao Y. Cellular metabolism on T-cell development and function. Int Rev Immunol 2015;34:19-33.

10. Shushimita S, de Bruijn MJ, de Bruin RW, IJ JN, Hendriks RW, Dor FJ. Dietary restriction and fasting arrest B and T cell development and increase mature $\mathrm{B}$ and $\mathrm{T}$ cell numbers in bone marrow. PLoS ONE 2014;9:e87772.

11. Hangen E, Feraud O, Lachkar S, Mou H, Doti N, Fimia GM, et al. Interaction between AIF and CHCHD4 regulates respiratory chain biogenesis. Mol Cell 2015;58:1001-14.

12. Klein JA, Longo-Guess CM, Rossmann MP, Seburn KL, Hurd RE, Frankel WN, et al. The harlequin mouse mutation downregulates apoptosis-inducing factor. Nature 2002;419:367-74.

13. Pospisilik JA, Knauf C, Joza N, Benit P, Orthofer M, Cani PD, et al. Targeted deletion of AIF decreases mitochondrial oxidative phosphorylation and protects from obesity and diabetes. Cell 2007;131:476-91.

14. Meyer K, Buettner S, Ghezzi D, Zeviani M, Bano D, Nicotera P. Loss of apoptosis-inducing factor critically affects MIA40 function. Cell Death Dis 2015;6:e1814.

15. Susin SA, Lorenzo HK, Zamzami N, Marzo I, Snow BE, Brothers $\mathrm{GM}$, et al. Molecular characterization of mitochondrial apoptosisinducing factor. Nature 1999;397:441-6.

16. Artus C, Boujrad H, Bouharrour A, Brunelle MN, Hoos S, Yuste $\mathrm{VJ}$, et al. AIF promotes chromatinolysis and caspase-independent programmed necrosis by interacting with histone $\mathrm{H} 2 \mathrm{AX}$. EMBO J 2010;29:1585-99.

17. Baritaud M, Cabon L, Delavallee L, Galan-Malo P, Gilles ME, Brunelle-Navas MN, et al. AIF-mediated caspaseindependent necroptosis requires ATM and DNA-PK-induced histone H2AX Ser139 phosphorylation. Cell Death Dis 2012;3: e390.

18. Cabon L, Galan-Malo P, Bouharrour A, Delavallee L, BrunelleNavas MN, Lorenzo HK, et al. BID regulates AIF-mediated caspase-independent necroptosis by promoting BAX activation. Cell Death Differ 2012;19:245-56.

19. Cregan SP, Dawson VL, Slack RS. Role of AIF in caspasedependent and caspase-independent cell death. Oncogene 2004; 23:2785-96.

20. Delavallee L, Cabon L, Galan-Malo P, Lorenzo HK, Susin SA. AIF-mediated caspase-independent necroptosis: a new chance for targeted therapeutics. IUBMB Life 2011;63:221-32.

21. Yu SW, Wang H, Poitras MF, Coombs C, Bowers WJ, Federoff $\mathrm{HJ}$, et al. Mediation of poly(ADP-ribose) polymerase-1-dependent cell death by apoptosis-inducing factor. Science 2002;297: 259-63.

22. Shen SM, Guo M, Xiong Z, Yu Y, Zhao XY, Zhang FF et al. AIF inhibits tumor metastasis by protecting PTEN from oxidation. EMBO Rep 2015; 16: 1563-1580.

23. Ghezzi D, Sevrioukova I, Invernizzi F, Lamperti C, Mora M, D'Adamo P, et al. Severe X-linked mitochondrial encephalomyopathy associated with a mutation in apoptosis-inducing factor. Am J Hum Genet 2010;86:639-49.

24. Berger I, Ben-Neriah Z, Dor-Wolman T, Shaag A, Saada A, Zenvirt S, et al. Early prenatal ventriculomegaly due to an AIFM1 mutation identified by linkage analysis and whole exome sequencing. Mol Genet Metab 2011;104:517-20.

25. Diodato D, Tasca G, Verrigni D, D’Amico A, Rizza T, Tozzi G et al. A novel AIFM1 mutation expands the phenotype to an infantile motor neuron disease. Eur J Hum Genet 2016; 24: 463-466.

26. Srivastava S, Banerjee H, Chaudhry A, Khare A, Sarin A, George $\mathrm{A}$, et al. Apoptosis-inducing factor regulates death in peripheral T cells. J Immunol 2007;179:797-803.

27. Banerjee H, Das A, Srivastava S, Mattoo HR, Thyagarajan K, Khalsa JK, et al. A role for apoptosis-inducing factor in $\mathrm{T}$ cell development. J Exp Med 2012;209:1641-53.

28. Milasta S, Dillon CP, Sturm OE, Verbist KC, Brewer TL, Quarato $\mathrm{G}$, et al. Apoptosis-inducing-factor-dependent mitochondrial function is required for $\mathrm{T}$ cell but Not B cell function. Immunity 2016; 44:88-102.

29. de Boer J, Williams A, Skavdis G, Harker N, Coles M, Tolaini M, et al. Transgenic mice with hematopoietic and lymphoid specific expression of Cre. Eur J Immunol 2003;33:314-25.

30. Hameyer D, Loonstra A, Eshkind L, Schmitt S, Antunes C, Groen $\mathrm{A}$, et al. Toxicity of ligand-dependent Cre recombinases and generation of a conditional Cre deleter mouse allowing mosaic recombination in peripheral tissues. Physiol Genomics 2007;31: $32-41$.

31. Lapuente-Brun E, Moreno-Loshuertos R, Acin-Perez R, LatorrePellicer A, Colas C, Balsa E, et al. Supercomplex assembly determines electron flux in the mitochondrial electron transport chain. Science 2013;340:1567-70.

32. Acin-Perez R, Enriquez JA. The function of the respiratory supercomplexes: the plasticity model. Biochim Biophys Acta 2014; 1837:444-50.

33. Dudkina NV, Kouril R, Peters K, Braun HP, Boekema EJ. Structure and function of mitochondrial supercomplexes. Biochim Biophys Acta 2010;1797:664-70.

34. Wang F, Flanagan J, Su N, Wang LC, Bui S, Nielson A, et al. RNAscope: a novel in situ RNA analysis platform for formalinfixed, paraffin-embedded tissues. J Mol Diagn 2012;14:22-9. 
35. Kang HC, Lee YM, Kim HD, Lee JS, Slama A. Safe and effective use of the ketogenic diet in children with epilepsy and mitochondrial respiratory chain complex defects. Epilepsia 2007;48: 82-8.

36. Wahlig JL, Bales ES, Jackman MR, Johnson GC, McManaman JL, Maclean PS. Impact of high-fat diet and obesity on energy balance and fuel utilization during the metabolic challenge of lactation. Obesity (Silver Spring) 2012;20:65-75.

37. Fox CJ, Hammerman PS, Thompson CB. Fuel feeds function: energy metabolism and the T-cell response. Nat Rev Immunol 2005;5:844-52.

38. Weinberg SE, Sena LA, Chandel NS. Mitochondria in the regulation of innate and adaptive immunity. Immunity 2015;42: 406-17.

39. Walker MA, Volpi S, Sims KB, Walter JE, Traggiai E. Powering the immune system: mitochondria in immune function and deficiency. J Immunol Res 2014;2014:164309.

40. Buck MD, O'Sullivan D, Pearce EL. T cell metabolism drives immunity. J Exp Med 2015;212:1345-60.

41. Joza N, Susin SA, Daugas E, Stanford WL, Cho SK, Li CY, et al. Essential role of the mitochondrial apoptosis-inducing factor in programmed cell death. Nature 2001;410:549-54.

42. Ishimura R, Martin GR, Ackerman SL. Loss of apoptosisinducing factor results in cell-type-specific neurogenesis defects. $\mathrm{J}$ Neurosci 2008;28:4938-48.

43. Benit P, Goncalves S, Dassa EP, Briere JJ, Rustin P. The variability of the harlequin mouse phenotype resembles that of human mitochondrial-complex I-deficiency syndromes. PLoS ONE 2008;3:e3208.

44. Cheung EC, Joza N, Steenaart NA, McClellan KA, Neuspiel M, McNamara S, et al. Dissociating the dual roles of apoptosisinducing factor in maintaining mitochondrial structure and apoptosis. Embo J 2006;25:4061-73.

45. Johnson MO, Rathmell JC. AIF Is "Always In Fashion" for T Cells. Immunity 2016;44:11-3.

46. Porritt HE, Rumfelt LL, Tabrizifard S, Schmitt TM, ZunigaPflucker JC, Petrie HT. Heterogeneity among DN1 prothymocytes reveals multiple progenitors with different capacities to generate $\mathrm{T}$ cell and non-T cell lineages. Immunity 2004;20:735-45.

47. Milicevic NM, Nohroudi K, Milicevic Z, Hedrich HJ, Westermann J. T cells are required for the peripheral phase of B-cell maturation. Immunology 2005;116:308-17.

48. Sena LA, Chandel NS. Physiological roles of mitochondrial reactive oxygen species. Mol Cell 2012;48:158-67.

49. Tothova Z, Kollipara R, Huntly BJ, Lee BH, Castrillon DH, Cullen DE, et al. FoxOs are critical mediators of hematopoietic stem cell resistance to physiologic oxidative stress. Cell 2007;128: 325-39.

50. van den Berg SM, Seijkens TT, Kusters PJ, Beckers L, den Toom $\mathrm{M}$, Smeets E, et al. Diet-induced obesity in mice diminishes hematopoietic stem and progenitor cells in the bone marrow. FASEB J 2016;30:1779-88.

51. Liu J, Cao L, Chen J, Song S, Lee IH, Quijano C, et al. Bmi1 regulates mitochondrial function and the DNA damage response pathway. Nature 2009;459:387-92.

52. Case AJ, McGill JL, Tygrett LT, Shirasawa T, Spitz DR, Waldschmidt TJ, et al. Elevated mitochondrial superoxide disrupts normal $\mathrm{T}$ cell development, impairing adaptive immune responses to an influenza challenge. Free Radic Biol Med 2011;50:448-58.

53. Schiff M, Benit P, Jacobs HT, Vockley J, Rustin P. Therapies in inborn errors of oxidative metabolism. Trends Endocrinol Metab 2012;23:488-95.

54. Juntilla MM, Wofford JA, Birnbaum MJ, Rathmell JC, Koretzky GA. Akt1 and Akt2 are required for alphabeta thymocyte survival and differentiation. Proc Natl Acad Sci USA 2007;104:12105-10.

55. Cao Y, Li H, Liu H, Zheng C, Ji H, Liu X. The serine/threonine kinase LKB1 controls thymocyte survival through regulation of AMPK activation and Bcl-XL expression. Cell Res 2010;20: 99-108.

56. Tamas P, Macintyre A, Finlay D, Clarke R, Feijoo-Carnero C, Ashworth A, et al. LKB1 is essential for the proliferation of T-cell progenitors and mature peripheral T cells. Eur J Immunol 2010; 40:242-53.

57. MacIver NJ, Michalek RD, Rathmell JC. Metabolic regulation of T lymphocytes. Annu Rev Immunol 2013;31:259-83.

58. Tcherkez G, Mahe A, Gauthier P, Mauve C, Gout E, Bligny R, et al. In folio respiratory fluxomics revealed by $13 \mathrm{C}$ isotopic labeling and H/D isotope effects highlight the noncyclic nature of the tricarboxylic acid "cycle" in illuminated leaves. Plant Physiol 2009;151:620-30.

59. Zhang J, Nuebel E, Wisidagama DR, Setoguchi K, Hong JS, Van Horn CM, et al. Measuring energy metabolism in cultured cells, including human pluripotent stem cells and differentiated cells. Nat Protoc 2012;7:1068-85.

60. Miller CL, Dykstra B, Eaves CJ. Characterization of mouse hematopoietic stem and progenitor cells. Curr Protoc Immunol 2008; Chapter 22: Unit 22B 2.

\section{Affiliations}

\section{Lauriane Cabon $^{1,2} \cdot$ Audrey Bertaux $^{1,2} \cdot$ Marie-Noëlle Brunelle-Navas $^{1,2} \cdot$ Ivan Nemazanyy $^{3} \cdot$ Laurianne Scourzic $^{4}$.

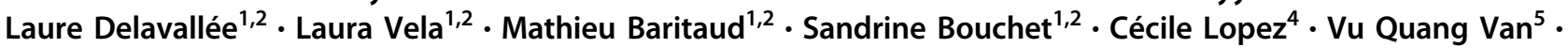 Kevin Garbin ${ }^{2,6} \cdot$ Danielle Chateau $^{2,6} \cdot$ Françoise Gilard $^{7} \cdot$ Marika Sarfati $^{5} \cdot$ Thomas Mercher $^{4} \cdot$ Olivier A. Bernard ${ }^{4}$. Santos A. Susin $\mathbb{D}^{1,2}$}

1 Cell Death and Drug Resistance in Lymphoproliferative Disorders Team, Centre de Recherche des Cordeliers, INSERM UMRS 1138, Paris, France

2 Sorbonne Universités, Université Pierre et Marie Curie, Paris, France

3 Université Paris Descartes, Sorbonne Paris Cité, Paris, France

4 INSERM U1170, Institut Gustave Roussy, Villejuif, France. Université Paris-Sud/Paris Saclay, Orsay, France
5 Immunoregulation Laboratory, Centre de Recherche du Centre Hospitalier de l'Université de Montréal (CRCHUM), Montréal, QC, Canada

6 Intestine: Nutrition, Barrier, and Diseases Team, Centre de Recherche des Cordeliers, INSERM UMRS 1138, Paris, France

7 Institute of Plant Sciences Paris-Saclay (IPS2), UMR 9213/ UMR1403, CNRS, INRA, Université d'Evry, Université ParisDiderot, Université Paris-Sud/Paris Saclay, Orsay, France 